\title{
A Comparison between the Reduced Differential Transform Method and Perturbation-Iteration Algorithm for Solving Two-Dimensional Unsteady Incompressible Navier-Stokes Equations
}

\author{
Abdul-Sattar J. Al-Saif, Assma J. Harfash \\ Department of Mathematics, College of Education for Pure Science, University of Basrah, Basra, Iraq \\ Email: sattaralsaif@yahoo.com, assmaj1974@yahoo.com
}

How to cite this paper: Al-Saif, A.-S.J. and Harfash, A.J. (2018) A Comparison between the Reduced Differential Transform Method and Perturbation-Iteration Algorithm for Solving Two-Dimensional Unsteady Incompressible Navier-Stokes Equations. Journal of Applied Mathematics and Physics, 6, 2518-2543.

https://doi.org/10.4236/jamp.2018.612211

Received: November 12, 2018

Accepted: December 9, 2018

Published: December 12, 2018

Copyright $\odot 2018$ by authors and Scientific Research Publishing Inc. This work is licensed under the Creative Commons Attribution International License (CC BY 4.0).

http://creativecommons.org/licenses/by/4.0/

\begin{abstract}
In this work, approximate analytical solutions to the lid-driven square cavity flow problem, which satisfied two-dimensional unsteady incompressible Navier-Stokes equations, are presented using the kinetically reduced local Navier-Stokes equations. Reduced differential transform method and perturbation-iteration algorithm are applied to solve this problem. The convergence analysis was discussed for both methods. The numerical results of both methods are given at some Reynolds numbers and low Mach numbers, and compared with results of earlier studies in the review of the literatures. These two methods are easy and fast to implement, and the results are close to each other and other numerical results, so it can be said that these methods are useful in finding approximate analytical solutions to the unsteady incompressible flow problems at low Mach numbers.
\end{abstract}

\section{Keywords}

Unsteady Incompressible Viscous Flows, Reduced Differential Transform Method, Perturbation-Iteration Algorithm

\section{Introduction}

Fluid flow is one of the most important engineering phenomena that have received widespread attention in theoretical and practical scientific research. Many of these studies focus on simulated mathematical models which represent these phenomena. Therefore, the equations of Navier-Stokes, which are the basic 
model for describing the movement of fluid, have received considerable attention from researchers to find their analytical and numerical solutions.

In this work, unsteady viscous incompressible flows characterized by two-dimensional Navier-Stokes equations are studied. The non-dimensional momentum and continuity equations have the following form

$$
\begin{aligned}
& u_{t}=-\left(u u_{x}+v u_{y}+p_{x}\right)+\frac{1}{R e}\left(u_{x x}+u_{y y}\right), \\
& v_{t}=-\left(u v_{x}+v v_{y}+p_{y}\right)+\frac{1}{R e}\left(v_{x x}+v_{y y}\right),
\end{aligned}
$$

and

$$
u_{x}+v_{y}=0
$$

where $t$ is the physical time, $u(x, y, t)$ and $v(x, y, t)$ are the fluid velocity components, $p(x, y, t)$ is the pressure, and Re is the Reynolds number. Since, the Navier-Stokes equations are nonlinear partial differential equations and there is no explicit equation for calculating pressure, these equations are difficult to solve, so many studies have suggested the alternative thermodynamic description of incompressible fluid flows. One of these alternative formulas is the kinetically reduced local Navier-Stokes (KRLNS) equations [1] [2] [3] [4] [5] which is obtained by replacing the pressure by

$$
p=g+\frac{u^{2}+v^{2}}{2}
$$

and the continuity equation by

$$
g_{t}=-\frac{1}{(M a)^{2}}\left(u_{x}+v_{y}\right)+\frac{1}{R e}\left(g_{x x}+g_{y y}\right),
$$

where $M a$ is the Mach number and $g(x, y, t)$ is the grand potential. The time scale in INS equations is related to that of KRLNS equations; $t_{K R L N S}(\tau)=M a \times t_{N S}$. Then, the system of equations of KRLNS has the following form

$$
\begin{aligned}
& u_{t}=-\left(2 u u_{x}+v v_{x}+v u_{y}+g_{x}\right)+\frac{1}{R e}\left(u_{x x}+u_{y y}\right), \\
& v_{t}=-\left(u v_{x}+u u_{y}+2 v v_{y}+g_{y}\right)+\frac{1}{R e}\left(v_{x x}+v_{y y}\right), \\
& g_{t}=-\frac{1}{(M a)^{2}}\left(u_{x}+v_{y}\right)+\frac{1}{R e}\left(g_{x x}+g_{y y}\right) .
\end{aligned}
$$

The KRINS equations suggested in [1] of the reduced equations for the grand potential and the fluid momentum were derived from the compressible Navier-Stokes equations in order to present the thermodynamic description of incompressible fluid flows at low Mach numbers. The two-dimensional KRLNS system is simplified and compared with a Chorin's artificial compressibility method for steady state computation of flow in two-dimensional lid-driven cavity and Taylor-Green vortex flow in [2]. In [3], KRLNS equations were applied to two-dimensional simulation of doubly periodic shear layers and decaying ho- 
mogeneous isotropic turbulence, where the central difference scheme is used for the spatial discrimination and four stage. Runge-Kutta method is utilized for the time integration. High order approach of the KRLNS equations was applied to two-dimensional numerical simulations of Womersley problem, doubly periodic shear layers and three-dimensional decaying homogeneous isotropic turbulence in [4] [5].

The lid-driven cavity problem refers to the flow in a box cavity with no-slip at the walls, one or more which move at constant speed. It has been used extensively as a benchmark case for the study of computational methods to solve Navier-Stokes equations, because the simplicity of its geometry and boundary conditions. Numerous literature studies have offered the solutions for this problem by using the different numerical methods in rectangular or square cavities. For example, in [6], the implicit cell-vertex finite volume method was described to solve the steady and unsteady two-dimensional lid-driven cavity problem at high Reynolds numbers. In [7], Chebyshev-collocation method in space is introduced with Adams-Bashforth backward-Euler scheme for the time integration to calculate the solution of three-dimensional lid-driven cavity flows. The finite element scheme based on the Galerkin method of weighted residuals of unsteady laminar mixed convection heat transfer in a lid driven cavity is performed in [8]. The vorticity-stream formulation of the Navier-Stokes equation with the strong-stability-preserving Runge-Kutta (SSPRK $(5,4)$ ) scheme in very fine grid mesh was used for solving lid driven cavity at high Reynolds number in [9]. For the problem of flow inside a square cavity with constant velocity, the finite volume method with numerical approximations of second-order accuracy and multiple Richardson extrapolations is utilized in [10]. The compact finite difference approximation is developed for non-uniform orthogonal Cartesian grids in [11] for solving the stream function-velocity formulation of the steady two dimensional incompressible lid-driven square cavity flow problem. The numerical simulations of two-dimensional fluid flow and heat transfer in a four-sided lid-driven rectangular domain have been preformed in [12], where the quadratic upstream interpolation for convective kinematics (QUICK) scheme of finite volume methods was used and semi-implicit method for pressure linked equations (SIMPLE algorithm) was adopted to compute the numerical solutions of the flow variables.

The main aim of this study is to obtain the approximate analytical solutions for two-dimensional lid-driven square cavity flow problem, since most of the research focused on the numerical solutions for this problem. Reduced differential transform method (RDTM) and perturbation-iteration algorithm (PIA) are used for this purpose for several reasons. The first reason is that both methods have not previously been applied to resolve this problem. Secondly, these methods can directly be applied to KRLNS equations. Moreover, these methods can reduce the size of the calculations and at the same time maintain the accuracy of the numerical solution.

We have organized this paper into seven sections, of which this introduction 
is the first. In Section 2 and 3, we describe the reduced differential transform method and perturbation-iteration algorithm, and applied them to KRLNS equations. We derived the condition of convergence for both methods (Section 4). We then present the approximate analytical solutions for two-dimensional lid-driven cavity flow, which are obtained by applying differential transform method and perturbation-iteration algorithm (Section 5). Next, we introduce the numerical results and compare these results with other works (Section 6). The last Section summarizes the major findings of this study.

\section{Reduced Differential Transform Method (RDTM)}

The RDTM was first introduced by Keskin [13]. It is an iterative procedure based on the use of the Taylor series solution of differential equations. It has been successfully applied to solve various nonlinear partial differential equations [13]-[27]. Since it does not require any parameter, discretization, linearization or small perturbations, thus it reduces the size of computations and can be easily used. The RDTM was used for solving the generalized Korteweg-de Vries equation [14], the fractional Benney-Lin equation [15], the Wu-Zhang equation [16], the equal width wave equation and the inviscid Burgers equation [17], the Sine-Gordon equation [18], the Burgers and Huxley equations [19], the time-fractional telegraph equation [20], the generalized Drinfeld-Sokolov equations and Kaup-Kupershmidt equation [21], the Zakharov-Kuznetsov equations [22], the heat-like equations [23], the coupled Ramani equations [25], two integral members of nonlinear Kadomtsev-Petviashvili hierarchy equations [26], and the second order hyperbolic telegraph equation [27]. Few studies have been applied RDTM to solve the Navier-Stocks equations, which is one of the reasons for choosing it as a method for solving the lid-driven cavity flow.

In this section, we give some properties of the $(2+1)$-dimensional RDTM [16] [18] [20] [22] [23] [24] [26] [27] which is used to find the approximate solutions to two-dimensional Navier-Stokes equations. Consider $X=(x, y)$ be a vector, if $u(X, t)$ is analytic function and continuously differentiable with respect to time $t$ and space in the domain of interest. Then, let

$$
U_{k}(X)=\frac{1}{k !}\left[\frac{\partial^{k}}{\partial t^{k}} u(X, t)\right]_{t=0},
$$

is the $t$-dimensional spectrum function of $u(X, t)$ which is the transformed function. The reduced differential inverse transform of $U_{k}(X)$ is defined as

$$
u(X, t)=\sum_{k=0}^{\infty} U_{k}(X) t^{k}
$$

from Equation (2.1) and Equation (2.2), we can conclude that

$$
u(X, t)=\sum_{k=0}^{\infty} \frac{1}{k !}\left[\frac{\partial^{k}}{\partial t^{k}} u(X, t)\right]_{t=0} t^{k} .
$$

The fundamental mathematical operations performed by RDTM are readily obtained and listed in Table 1. 
Table 1. Reduced differential transformation.

\begin{tabular}{cc}
\hline Functional form & Transformed form \\
\hline$w(X, t)=u(X, t) v(X, t)$ & $W_{k}(X)=U_{k}(X) V_{k}(X)$ \\
$w(X, t)=\alpha \dot{u}(X, t)$ & $W_{k}(X)=\alpha \dot{U}_{k}(X), \alpha$ is constant \\
$w(X, t)=u(X, t) \dot{v}(X, t)$ & $W_{k}(X)=\sum_{i=0}^{k} U_{i}(X, t) \dot{V}_{k-i}(X, t)$ \\
$w(x, t)=\frac{\partial^{r}}{\partial t^{r}} u(X, t)$ & $W_{k}(X)=(k+1) \cdots(k+r) \dot{U}_{k}(X)=\frac{(k+r) !}{k !} \dot{U}_{k}(X)$ \\
$w(X, t)=\frac{\partial^{r_{1}+r_{2}+\cdots+r_{n}}}{\partial x_{1}^{r} \partial x_{2}^{r_{2}} \cdots \partial x_{n}^{r_{n}}} u(X, t)$ & $W_{k}(x)=\frac{\partial^{r_{1}+r_{2}+\cdots+r_{n}}}{\partial x_{1}^{r_{1}} \partial x_{2}^{r_{2}} \cdots \partial x_{n}^{r_{n}}} U_{k}(X)$ \\
\hline
\end{tabular}

In order to apply this method with KRLNS equations to find approximate analytical solutions for INS equations, we suppose that $X=(x, y), \mathbf{u}=(u, v)$ and $\mathbf{U}_{k}=\left(U_{k}, V_{k}\right)$, where $u(X, t)$ and $v(X, t)$ are the fluid velocity components in the $x$ and $y$ directions, and $U_{k}(X), V_{k}(X)$ and $G_{k}(X)$ are t-dimensional spectrum functions of $u(X, t), \quad v(X, t)$ and $g(X, t)$ respectively. Then, we have

$$
\begin{aligned}
& (k+1) U_{k+1}(X) \\
= & -\left(2 A_{k}+B_{k}+C_{k}+\left(G_{k}(X)\right)_{x}-\frac{1}{R e}\left(\left(U_{k}(X)\right)_{x x}+\left(U_{k}(X)\right)_{y y}\right)\right), \\
& (k+1) V_{k+1}(X) \\
& =-\left(D_{k}+E_{k}+2 F_{k}+\left(G_{k}(X)\right)_{y}-\frac{1}{R e}\left(\left(V_{k}(X)\right)_{x x}+\left(V_{k}(X)\right)_{y y}\right)\right), \\
( & +1) G_{k+1}(X) \\
= & -\left(\frac{1}{(M a)^{2}}\left(\left(U_{k}(X)\right)_{x}+\left(V_{k}(X)\right)_{y}\right)-\frac{1}{\operatorname{Re}}\left(\left(G_{k}(X)\right)_{x x}+\left(G_{k}(X)\right)_{y y}\right)\right),
\end{aligned}
$$

such that

$$
\begin{array}{ll}
A_{k}=\sum_{i=0}^{k} U_{i}(X)\left(U_{k-i}(X)\right)_{x}, & B_{k}=\sum_{i=0}^{k} V_{i}(X)\left(V_{k-i}(X)\right)_{x}, \\
C_{k}=\sum_{i=0}^{k} V_{i}(X)\left(U_{k-i}(X)\right)_{y}, & D_{k}=\sum_{i=0}^{k} U_{i}(X)\left(V_{k-i}(X)\right)_{x}, \\
E_{k}=\sum_{i=0}^{k} U_{i}(X)\left(U_{k-i}(X)\right)_{y}, & F_{k}=\sum_{i=0}^{k} V_{i}(X)\left(V_{k-i}(X)\right)_{y},
\end{array}
$$

where $k=0,1,2,3, \cdots, U_{0}(X)=u(X, 0), V_{0}(X)=v(X, 0)$ and $G_{0}(X)=g(X, 0)$. Then the exact solution is obtained as follows:

$$
\begin{gathered}
u(X, \tau)=\lim _{n \rightarrow \infty} u_{n}(X, \tau), \\
v(X, \tau)=\lim _{n \rightarrow \infty} v_{n}(X, \tau), \\
g(X, \tau)=\lim _{n \rightarrow \infty} g_{n}(X, \tau),
\end{gathered}
$$

where 


$$
u_{n}(X, \tau)=\sum_{k=0}^{n} U_{k}(X) \tau^{k}, v_{n}(X, \tau)=\sum_{k=0}^{n} V_{k}(X) \tau^{k}, g_{n}(X, \tau)=\sum_{k=0}^{n} G_{k}(X) \tau^{k} .
$$

This approach is referred to by (KRDTM) in this paper.

\section{Perturbation-Iteration Algorithm (PIA)}

Perturbation methods are important analytical methods which have been used to construct approximate analytical solutions of algebraic equations, differential equations, and integro-differential equations. The main limitation of using the perturbation methods is to install a small auxiliary parameter in the equation. For this reason, the solutions of these methods are restricted by validity range of physical parameters, so many of perturbation techniques have been suggested by several authors. PIA is one of the techniques which was proposed by Pakdemirli and Boyac in [28], and used a combination of perturbation expansions and Taylor series expansions to construct an iteration scheme for using to generate root finding algorithms. It is applied by many authors to get the approximate analytical solution for differential equations. In [29], PIA was applied to obtain the solution of Bratu-type equations. In [30], PIA was utilized to find the solution first order differential equations. This algorithm was tested on three nonlinear heat equations in [31]. Moreover, PIA was generalized to an arbitrary number of first-order coupled equations in [32]. It was applied to Fredholm and Volterra integral equations in [33]. Also, in [34], PIA was proposed for solving the Riccati differential equation. It was developed in [35] to obtain the solutions of Lotka-Volterra differential equations. In [36], some types of fractional differential equation systems were solved by using this method. PIA with Laplace transform method was combined in [37] to solve Newell-Whitehead-Segel equations. In [38], PIA is used for solving the fractional Zakharov-Kuznetsov equation and compared with the residual power series method. By reviewing the previous literature, we have not found any research that has used this method to find a solution to the two-dimensional lid-driven cavity flow problem and which is an important reason to use this method to solve this problem.

In general, PIA is obtained by taking different numbers of terms in the perturbation expansions and different order of correction terms in the Taylor series expansions. Therefore, the perturbation-iteration algorithm is called PIA $(m, n)$ where the $m$ is the number of the correction terms in the perturbation expansion and $n$ is the highest order derivative term in the Taylor series such that $m$ should always be less than or equal to $n$.

To obtain approximate analytical solutions for two-dimensions Navier-Stokes equations, PIA $(1,1)$ will be applied to KRLNS equations and which will be referred to this article by (KPIA). Firstly, we write Equation (1.5) as follows:

$$
\begin{aligned}
& F_{1}\left(u, v, u_{t}, u_{x}, u_{y}, v_{x}, g_{x}, u_{x x}, u_{y y}, \epsilon\right) \\
& =u_{t}+\epsilon\left(2 u u_{x}+v v_{x}+v u_{y}+g_{x}-\frac{1}{R e}\left(u_{x x}+u_{y y}\right)\right),
\end{aligned}
$$




$$
\begin{aligned}
& F_{2}\left(u, v, v_{t}, u_{y}, v_{x}, v_{y}, g_{y}, v_{x x}, v_{y y}, \epsilon\right) \\
& =v_{t}+\epsilon\left(u v_{x}+u u_{y}+2 v v_{y}+g_{y}-\frac{1}{R e}\left(v_{x x}+v_{y y}\right)\right), \\
& F_{3}\left(g_{t}, u_{x}, v_{y}, g_{x x}, g_{y y}, \epsilon\right) \\
& =g_{t}+\epsilon\left(\frac{1}{(M a)^{2}}\left(u_{x}+v_{y}\right)-\frac{1}{R e}\left(g_{x x}+g_{y y}\right)\right),
\end{aligned}
$$

where $\epsilon$ is a small perturbation parameter. Secondly, we define the following perturbation expansions with only one correction term:

$$
\begin{aligned}
& u_{n+1}=u_{n}+\epsilon\left(u_{c}\right)_{n}, \\
& v_{n+1}=v_{n}+\epsilon\left(v_{c}\right)_{n}, \\
& g_{n+1}=g_{n}+\epsilon\left(g_{c}\right)_{n},
\end{aligned}
$$

where $n$ represents the $n_{-}$th iteration and $u_{c}, v_{c}$ and $g_{c}$ are the correction terms in the perturbation expansion. Thirdly, by replacing (3.2) into (3.1) and writing in the Taylor series expansion for first order derivative terms about $\epsilon=0$, yields

$$
\begin{aligned}
& F_{1}\left(u_{n}, v_{n},\left(u_{n}\right)_{t},\left(u_{n}\right)_{x},\left(u_{n}\right)_{y},\left(v_{n}\right)_{x},\left(g_{n}\right)_{x},\left(u_{n}\right)_{x x},\left(u_{n}\right)_{y y}, 0\right) \\
& +\epsilon\left[F_{1 \epsilon}+F_{1 u_{n+1}}\left(u_{c}\right)_{n}+F_{1 v_{n+1}}\left(v_{c}\right)_{n}+F_{1\left(u_{n+1}\right)_{t}}\left(\left(u_{c}\right)_{n}\right)_{t}\right. \\
& +F_{1\left(u_{n+1}\right)_{x}}\left(\left(u_{c}\right)_{n}\right)_{x}+F_{1\left(u_{n+1}\right)_{y}}\left(\left(u_{c}\right)_{n}\right)_{y}+F_{1\left(v_{n+1}\right)_{x}}\left(\left(v_{c}\right)_{n}\right)_{x} \\
& \left.+F_{1\left(g_{n+1}\right)_{x}}\left(\left(g_{c}\right)_{n}\right)_{x}+F_{1\left(u_{n+1}\right)_{x x}}\left(\left(u_{c}\right)_{n}\right)_{x x}+F_{1\left(u_{n+1}\right)_{y y}}\left(\left(u_{c}\right)_{n}\right)_{y y}\right]=0, \\
& F_{2}\left(u_{n}, v_{n},\left(v_{n}\right)_{t},\left(u_{n}\right)_{y},\left(v_{n}\right)_{x},\left(v_{n}\right)_{y},\left(g_{n}\right)_{y},\left(v_{n}\right)_{x x},\left(v_{n}\right)_{y y}, 0\right) \\
& +\epsilon\left[F_{2 \epsilon}+F_{2 u_{n+1}}\left(u_{c}\right)_{n}+F_{2 v_{n+1}}\left(v_{c}\right)_{n}+F_{2\left(v_{n+1}\right)_{t}}\left(\left(v_{c}\right)_{n}\right)_{t}\right. \\
& +F_{2\left(u_{n+1}\right)_{y}}\left(\left(u_{c}\right)_{n}\right)_{y}+F_{2\left(v_{n+1}\right)_{x}}\left(\left(v_{c}\right)_{n}\right)_{x}+F_{2\left(v_{n+1}\right)_{y}}\left(\left(v_{c}\right)_{n}\right)_{y} \\
& \left.+F_{2\left(g_{n+1}\right)_{y}}\left(\left(g_{c}\right)_{n}\right)_{y}+F_{2\left(v_{n+1}\right)_{x x}}\left(\left(v_{c}\right)_{n}\right)_{x x}+F_{2\left(v_{n+1}\right)_{y y}}\left(\left(v_{c}\right)_{n}\right)_{y y}\right]=0, \\
& F_{3}\left(\left(g_{n}\right)_{t},\left(u_{n}\right)_{x},\left(v_{n}\right)_{y},\left(g_{n}\right)_{x x},\left(g_{n}\right)_{y y}, 0\right) \\
& +\epsilon\left[F_{3 \epsilon}+F_{3\left(g_{n+1}\right)_{t}}\left(\left(g_{c}\right)_{n}\right)_{t}+F_{3\left(u_{n+1}\right)_{x}}\left(\left(u_{c}\right)_{n}\right)_{x}+F_{3\left(v_{n+1}\right)_{y}}\left(\left(v_{c}\right)_{n}\right)_{y}\right. \\
& \left.+F_{3\left(g_{n+1}\right)_{x x}}\left(\left(g_{c}\right)_{n}\right)_{x x}+F_{3\left(g_{n+1}\right)_{y y}}\left(\left(g_{c}\right)_{n}\right)_{y y}\right]=0 .
\end{aligned}
$$

All derivatives in Equation (3.3) are evaluated at $\epsilon=0$ such that

$$
\begin{gathered}
F_{1}\left(u_{n}, v_{n},\left(u_{n}\right)_{t},\left(u_{n}\right)_{x},\left(u_{n}\right)_{y},\left(v_{n}\right)_{x},\left(g_{n}\right)_{x},\left(u_{n}\right)_{x x},\left(u_{n}\right)_{y y}, 0\right)=\left(u_{n}\right)_{t}, \\
F_{2}\left(u_{n}, v_{n},\left(v_{n}\right)_{t},\left(u_{n}\right)_{y},\left(v_{n}\right)_{x},\left(v_{n}\right)_{y},\left(g_{n}\right)_{y},\left(v_{n}\right)_{x x},\left(v_{n}\right)_{y y}, 0\right)=\left(v_{n}\right)_{t}, \\
F_{3}\left(\left(g_{n}\right)_{t},\left(u_{n}\right)_{x},\left(v_{n}\right)_{y},\left(g_{n}\right)_{x x},\left(g_{n}\right)_{y y}, 0\right)=\left(g_{n}\right)_{t}, \\
F_{1\left(u_{n+1}\right)_{t}}=F_{1\left(v_{n+1}\right)_{t}}=F_{3\left(g_{n+1}\right)_{t}}=1,
\end{gathered}
$$




$$
\begin{gathered}
F_{1 u_{n+1}}=F_{1 v_{n+1}}=F_{1\left(u_{n+1}\right)_{x}}=F_{1\left(u_{n+1}\right)_{y}}=F_{1\left(v_{n+1}\right)_{x}}=F_{1\left(u_{n+1}\right)_{x x}}=F_{1\left(u_{n+1}\right)_{y y}}=0, \\
F_{2 u_{n+1}}=F_{2 v_{n+1}}=F_{2\left(v_{n+1}\right)_{x}}=F_{2\left(v_{n+1}\right)_{y}}=F_{2\left(u_{n+1}\right)_{y}}=F_{2\left(v_{n+1}\right)_{x x}}=F_{2\left(v_{n+1}\right)_{y y}}=0, \\
F_{1\left(g_{n+1}\right)_{x}}=F_{2\left(g_{n+1}\right)_{y}}=F_{3\left(u_{n+1}\right)_{x}}=F_{3\left(v_{n+1}\right)_{y}}=F_{3\left(g_{n+1}\right)_{x x}}=F_{3\left(g_{n+1}\right)_{y y}}=0, \\
F_{1 \epsilon}=\left(2 u_{n}\left(u_{n}\right)_{x}+v_{n}\left(v_{n}\right)_{x}+v_{n}\left(u_{n}\right)_{y}+\left(g_{n}\right)_{x}-\frac{1}{R e}\left(\left(u_{n}\right)_{x x}+\left(u_{n}\right)_{y y}\right)\right), \\
F_{2 \epsilon}=\left(u_{n}\left(v_{n}\right)_{x}+u_{n}\left(u_{n}\right)_{y}+2 v_{n}\left(v_{n}\right)_{y}+\left(g_{n}\right)_{y}-\frac{1}{R e}\left(\left(v_{n}\right)_{x x}+\left(v_{n}\right)_{y y}\right)\right), \\
F_{3 \epsilon}=\left(\frac{1}{(M a)^{2}}\left(\left(u_{n}\right)_{x}+\left(v_{n}\right)_{y}\right)-\frac{1}{R e}\left(\left(g_{n}\right)_{x x}+\left(g_{n}\right)_{y y}\right)\right) .
\end{gathered}
$$

Finally, by substituting the above derivative in the formulas (3.3) and setting $\epsilon=1$ we obtain the following iteration equation formulas:

$$
\begin{gathered}
\left(\left(u_{c}\right)_{n}\right)_{t}=\left(u_{n}\right)_{t}-\left(2 u_{n}\left(u_{n}\right)_{x}+v_{n}\left(v_{n}\right)_{x}+v_{n}\left(u_{n}\right)_{y}+\left(g_{n}\right)_{x}-\frac{1}{R e}\left(\left(u_{n}\right)_{x x}+\left(u_{n}\right)_{y y}\right)\right), \\
\left(\left(v_{c}\right)_{n}\right)_{t}=\left(v_{n}\right)_{t}-\left(u_{n}\left(v_{n}\right)_{x}+u_{n}\left(u_{n}\right)_{y}+2 v_{n}\left(v_{n}\right)_{y}+\left(g_{n}\right)_{y}-\frac{1}{R e}\left(\left(v_{n}\right)_{x x}+\left(v_{n}\right)_{y y}\right)\right), \\
\left(\left(g_{c}\right)_{n}\right)_{t}=\left(g_{n}\right)_{t}-\left(\frac{1}{(M a)^{2}}\left(\left(u_{n}\right)_{x}+\left(v_{n}\right)_{y}\right)-\frac{1}{R e}\left(\left(g_{n}\right)_{x x}+\left(g_{n}\right)_{y y}\right)\right) .
\end{gathered}
$$

The calculations start with initial condition $u(x, y, 0), v(x, y, 0)$ and $g(x, y, 0)$ where these values are used as estimate values for $\left(u_{c}\right)_{0},\left(v_{c}\right)_{0}$ and $\left(g_{c}\right)_{0}$ in Equation (3.4), and then substitute the results of Equation (3.4) into Equation (3.2) to obtain $u_{1}, v_{1}$ and $g_{1}$ which are the solutions at the first iteration. So we can get $(n+1)$ iteration solutions by repeating this process and using the previous solution $n$ as an initial guess.

\section{Analysis of Convergence}

We now study the convergence analysis of the approximate analytical solutions which are computed from the application KRDTM and KPIA.

Let us consider the Hilbert space $H=L^{2}\left((a, b)^{2} \times[0, T]\right)$ as defined by

$$
u: H \rightarrow \mathbb{R} \text { with } \int_{(a, b)^{2} \times[0, T]} u^{2}(X, t) \mathrm{d} X \mathrm{~d} t<\infty,
$$

and the norm

$$
\|u\|^{2}=\int_{(a, b)^{2} \times[0, T]} u^{2}(X, t) \mathrm{d} X \mathrm{~d} t,
$$

where $X=(x, y)$. Defined as

$$
\mathbf{u}=(u, v, g): H^{3} \rightarrow \mathbb{R}^{3} \text { with } \int_{(a, b)^{2} \times[0, T]}\left(u^{2}(X, t)+v^{2}(X, t)+g^{2}(X, t)\right) \mathrm{d} X \mathrm{~d} t<\infty,
$$

such that $\|\mathbf{u}\|^{2}=\|u\|^{2}+\|v\|^{2}+\|g\|^{2}$.

We consider the KRINS equation in the following form

$$
\mathcal{L}(\mathbf{u}(X, \tau))=\mathcal{N}(\mathbf{u}(X, \tau))+\mathcal{R}(\mathbf{u}(X, \tau)),
$$


which is equivalent to the following formula

$$
\mathbf{u}(X, \tau)=\mathcal{F}\left(\mathbf{u}_{k}(X, \tau)\right),
$$

where $\mathcal{L}$ is the linear partial derivative with respect to $\tau, \mathcal{N}$ is a nonlinear operator, $\mathcal{R}$ is a linear operator, and $\mathcal{F}$ is a general nonlinear operator involving both linear and nonlinear terms.

Case 1: According to KRDTM, formula (4.1) can be written in the following form

$$
(k+1) \mathbf{U}_{k+1}(X)=\mathcal{N}\left(\mathbf{U}_{k}(X)\right)+\mathcal{R}\left(\mathbf{U}_{k}(X)\right),
$$

and the solutions

$$
\mathbf{u}(X, \tau)=\sum_{k=0}^{\infty} \mathbf{U}_{k}(X) \tau^{k}=\sum_{k=0}^{\infty} \mathcal{B}_{k},
$$

where $\mathcal{B}_{k}=\left(\mathcal{B}_{1 k}, \mathcal{B}_{2 k}, \mathcal{B}_{3 k}\right)$. It is noted that the solutions by KRDTM is equivalent to determining the sequence

$$
\begin{gathered}
\mathbf{S}_{0}=\mathbf{U}_{0}(X)=\mathcal{B}_{0}, \\
\mathbf{S}_{1}=\mathbf{U}_{0}(X)+\mathbf{U}_{1}(X) \tau=\mathcal{B}_{0}+\mathcal{B}_{1}, \\
\mathbf{S}_{2}=\mathbf{U}_{0}(X)+\mathbf{U}_{1}(X) \tau+\mathbf{U}_{2}(X) \tau^{2}=\mathcal{B}_{0}+\mathcal{B}_{1}+\mathcal{B}_{2}, \\
\vdots \\
\mathbf{S}_{n}=\sum_{k=0}^{n} \mathbf{U}_{k}(X) \tau^{k}=\sum_{k=0}^{n} \mathcal{B}_{k} .
\end{gathered}
$$

Case 2: To study the convergence of KPIA, we write the approximate solutions in different form. To do this, we define

$$
\begin{gathered}
\mathcal{B}_{0}=\left(\mathcal{B}_{10}, \mathcal{B}_{20}, \mathcal{B}_{30}\right)=(u(X, 0), v(X, 0), g(X, 0))=\mathbf{u}(X, 0), \\
\mathcal{B}_{n+1}=\left(\mathcal{B}_{1(n+1)}, \mathcal{B}_{2(n+1)}, \mathcal{B}_{3(n+1)}\right) \\
=\left(\left(u_{c}\right)_{n}(X, \tau),\left(v_{c}\right)_{n}(X, \tau),\left(g_{c}\right)_{n}(X, \tau)\right)=\left(\mathbf{u}_{c}\right)_{n}(X, \tau), \\
\mathbf{u}_{0}=\mathcal{B}_{0}=\mathbf{S}_{0}, \\
\mathbf{u}_{1}=\mathbf{u}_{0}+\epsilon\left(\mathbf{u}_{c}\right)_{0}=\mathcal{B}_{0}+\mathcal{B}_{1}=\mathbf{S}_{1}, \\
\mathbf{u}_{2}=\mathbf{u}_{1}+\epsilon\left(\mathbf{u}_{c}\right)_{1}=\mathcal{B}_{0}+\mathcal{B}_{1}+\mathcal{B}_{2}=\mathbf{S}_{2}, \\
\mathbf{u}_{3}=\mathbf{u}_{2}+\epsilon\left(\mathbf{u}_{c}\right)_{2}=\mathcal{B}_{0}+\mathcal{B}_{1}+\mathcal{B}_{2}+\mathcal{B}_{3}=\mathbf{S}_{3}, \\
\vdots \\
\mathbf{u}_{n}=\mathbf{u}_{n-1}+\epsilon\left(\mathbf{u}_{c}\right)_{n-1} \equiv \mathcal{B}_{0}+\mathcal{B}_{1}+\mathcal{B}_{2}+\mathcal{B}_{3}+\cdots+\mathcal{B}_{n}=\sum_{k=0}^{n} \mathcal{B}_{k}=\mathbf{S}_{n} .
\end{gathered}
$$

So the solutions, which are resulted from KPIA have the form

$$
\mathbf{u}(X, \tau)=\sum_{k=0}^{\infty} \mathbf{U}_{k}(X) \tau^{k}=\sum_{k=0}^{\infty} \mathcal{B}_{k} .
$$

such that $\mathbf{S}_{n+1}=\mathcal{F}\left(\mathbf{S}_{n}\right)$ for both cases. 
The sufficient condition for convergence of the series solution $\left\{\mathbf{S}_{n}\right\}_{0}^{\infty}$ is given in the following theorems.

Theorem 4.1. The series solution $\left\{\mathbf{S}_{n}=\left(R_{n}, S_{n}, T_{n}\right)\right\}_{0}^{\infty}$ converges whenever there is $\gamma$ such that $0<\gamma<1, \quad \gamma=\gamma_{1}+\gamma_{2}+\gamma_{3}$ and $\left\|\mathcal{B}_{i(k+1)}\right\| \leqslant \gamma_{i}\left\|\mathcal{B}_{i k}\right\|$.

Proof. Firstly, we show that $\left\{\mathbf{S}_{n}=\left(R_{n}, S_{n}, T_{n}\right)\right\}_{0}^{\infty}$ is a Cauchy sequence in the Hilbert space $H^{3}$. For this reason, we suppose that

$$
\begin{aligned}
& \left\|R_{n+1}-R_{n}\right\|=\left\|\mathcal{B}_{1(n+1)}\right\| \leqslant \gamma_{1}\left\|\mathcal{B}_{1 n}\right\| \leqslant \gamma_{1}^{2}\left\|\mathcal{B}_{1(n-1)}\right\| \leqslant \cdots \leqslant \gamma_{1}^{n+1}\left\|\mathcal{B}_{10}\right\|, \\
& \left\|S_{n+1}-S_{n}\right\|=\left\|\mathcal{B}_{2(n+1)}\right\| \leqslant \gamma_{2}\left\|\mathcal{B}_{2 n}\right\| \leqslant \gamma_{2}^{2}\left\|\mathcal{B}_{2(n-1)}\right\| \leqslant \cdots \leqslant \gamma_{2}^{n+1}\left\|\mathcal{B}_{20}\right\|, \\
& \left\|T_{n+1}-T_{n}\right\|=\left\|\mathcal{B}_{3(n+1)}\right\| \leqslant \gamma_{3}\left\|\mathcal{B}_{3 n}\right\| \leqslant \gamma_{3}^{2}\left\|\mathcal{B}_{3(n-1)}\right\| \leqslant \cdots \leqslant \gamma_{3}^{n+1}\left\|\mathcal{B}_{30}\right\| .
\end{aligned}
$$

Then, by using the triangle inequality, we find that

$$
\begin{aligned}
\left\|\mathbf{S}_{n}-\mathbf{S}_{m}\right\|= & \left\|\left(R_{n}, S_{n}, T_{n}\right)-\left(R_{m}, S_{m}, T_{m}\right)\right\|=\left\|\left(R_{n}-R_{m}, S_{n}-S_{m}, T_{n}-T_{m}\right)\right\| \\
\leqslant & \left\|R_{n}-R_{m}\right\|+\left\|S_{n}-S_{m}\right\|+\left\|T_{n}-T_{m}\right\| \\
\leqslant & \left\|R_{n}-R_{n-1}\right\|+\left\|R_{n-1}-R_{n-2}\right\|+\cdots+\left\|R_{m+1}-R_{m}\right\| \\
& +\left\|S_{n}-S_{n-1}\right\|+\left\|S_{n-1}-S_{n-2}\right\|+\cdots+\left\|S_{m+1}-S_{m}\right\| \\
& +\left\|T_{n}-T_{n-1}\right\|+\left\|T_{n-1}-T_{n-2}\right\|+\cdots+\left\|T_{m+1}-T_{m}\right\| \\
\leqslant & \left(\gamma_{1}^{n}+\gamma_{1}^{n-1}+\cdots+\gamma_{1}^{m+1}\right)\left\|\mathcal{B}_{10}\right\|+\left(\gamma_{2}^{n}+\gamma_{2}^{n-1}+\cdots+\gamma_{2}^{m+1}\right)\left\|\mathcal{B}_{20}\right\| \\
& +\left(\gamma_{3}^{n}+\gamma_{3}^{n-1}+\cdots+\gamma_{3}^{m+1}\right)\left\|\mathcal{B}_{30}\right\| \\
\leqslant & \left(\gamma^{n}+\gamma^{n-1}+\cdots+\gamma^{m+1}\right)\left(\left\|\mathcal{B}_{10}\right\|+\left\|\mathcal{B}_{20}\right\|+\left\|\mathcal{B}_{30}\right\|\right) \\
= & \gamma^{m+1}\left(\gamma^{n-m-1}+\gamma^{n-m-2}+\cdots+1\right)\left(\left\|\mathcal{B}_{10}\right\|+\left\|\mathcal{B}_{20}\right\|+\left\|\mathcal{B}_{30}\right\|\right) \\
\leqslant & \frac{\gamma^{m+1}}{1-\gamma}\left\|\mathcal{B}_{0}\right\|,
\end{aligned}
$$

since $\left\|\mathcal{B}_{0}\right\|<\infty$ and $0<\gamma<1$, we then have $\lim _{n, m \rightarrow \infty}\left\|\mathbf{S}_{n}-\mathbf{S}_{m}\right\|=0$. Thus, we conclude that $\left\{\mathbf{S}_{n}\right\}_{0}^{\infty}$ is a Cauchy sequence in the Hilbert space $H^{3}$, thus, the series solution $\left\{\mathbf{S}_{n}\right\}_{0}^{\infty}$ converges to some $\{\mathbf{S}\} \in H^{3}$.

Theorem 4.2. Let $\mathcal{F}=\left(\mathcal{F}_{1}, \mathcal{F}_{2}, \mathcal{F}_{3}\right)$ be a nonlinear operator satisfies Lipschitz condition from a Hilbert space $H^{\beta}$ into $H^{\beta}$ and $\mathbf{u}(X, \tau)$ be the exact solution of INS equations. If the series solution $\left\{\mathbf{S}_{n}\right\}_{0}^{\infty}$ converges, then it is converged to $\mathbf{u}(X, \tau)$.

Proof. Let $\mathbf{u}_{1}(X, \tau), \mathbf{u}_{2}(X, \tau)$, then we have

$$
\begin{aligned}
& \left\|\mathcal{F}\left(\mathbf{u}_{1}\right)-\mathcal{F}\left(\mathbf{u}_{2}\right)\right\| \\
& =\left\|\left(\mathcal{F}_{1}\left(\mathbf{u}_{1}\right), \mathcal{F}_{2}\left(\mathbf{u}_{1}\right), \mathcal{F}_{3}\left(\mathbf{u}_{1}\right)\right)-\left(\mathcal{F}_{1}\left(\mathbf{u}_{2}\right), \mathcal{F}_{2}\left(\mathbf{u}_{2}\right), \mathcal{F}_{3}\left(\mathbf{u}_{2}\right)\right)\right\| \\
& =\left\|\left(\mathcal{F}_{1}\left(\mathbf{u}_{1}\right)-\mathcal{F}_{1}\left(\mathbf{u}_{2}\right), \mathcal{F}_{2}\left(\mathbf{u}_{1}\right)-\mathcal{F}_{2}\left(\mathbf{u}_{2}\right), \mathcal{F}_{3}\left(\mathbf{u}_{1}\right)-\mathcal{F}_{3}\left(\mathbf{u}_{2}\right)\right)\right\| \\
& \leqslant\left\|\mathcal{F}_{1}\left(\mathbf{u}_{1}\right)-\mathcal{F}_{1}\left(\mathbf{u}_{2}\right)\right\|+\left\|\mathcal{F}_{2}\left(\mathbf{u}_{1}\right)-\mathcal{F}_{2}\left(\mathbf{u}_{2}\right)\right\|+\left\|\mathcal{F}_{3}\left(\mathbf{u}_{1}\right)-\mathcal{F}_{3}\left(\mathbf{u}_{2}\right)\right\| \\
& \leqslant \alpha_{1}\left\|\mathbf{u}_{1}-\mathbf{u}_{2}\right\|+\alpha_{2}\left\|\mathbf{u}_{1}-\mathbf{u}_{2}\right\|+\alpha_{3}\left\|\mathbf{u}_{1}-\mathbf{u}_{2}\right\| \\
& =\left(\alpha_{1}+\alpha_{2}+\alpha_{3}\right)\left\|\mathbf{u}_{1}-\mathbf{u}_{2}\right\|=\alpha\left\|\mathbf{u}_{1}-\mathbf{u}_{2}\right\| .
\end{aligned}
$$

Therefore, from the Banach fixed-point theorem, there is a unique solution of the problem (4.1). Now we have to prove that $\left\{\mathbf{S}_{n}\right\}_{0}^{\infty}$ converges to $\mathbf{u}(X, \tau)$ 


$$
\begin{aligned}
\mathbf{u}(X, \tau) & =\mathcal{F}(\mathbf{u}(X, \tau))=\mathcal{F}\left(\sum_{k=0}^{\infty} \mathcal{B}_{k}\right)=\mathcal{F}\left(\lim _{n \rightarrow \infty} \sum_{k=0}^{n} \mathcal{B}_{k}\right) \\
& =\lim _{n \rightarrow \infty} \mathcal{F}\left(\sum_{k=0}^{n} \mathcal{B}_{k}\right)=\lim _{n \rightarrow \infty} \mathcal{F}\left(\mathbf{S}_{n}\right)=\lim _{n \rightarrow \infty} \mathbf{S}_{n+1}=\mathbf{S} .
\end{aligned}
$$

Definition 4.1. For $i=1,2,3$ and $k \in \mathbb{N} \cup\{0\}$, we define

$$
\gamma_{i k}= \begin{cases}\frac{\left\|\mathcal{B}_{i(k+1)}\right\|}{\left\|\mathcal{B}_{i k}\right\|}, & \left\|\mathcal{B}_{i k}\right\| \neq 0, \\ 0, & \left\|\mathcal{B}_{i k}\right\|=0 .\end{cases}
$$

then we can say that the series approximate solutions $\left\{\mathbf{S}_{n}\right\}_{0}^{\infty}$ converges to the exact solution $\mathbf{u}(X, t)$ when $\gamma_{k}=\gamma_{1 k}+\gamma_{2 k}+\gamma_{3 k}$ and $0<\gamma_{k}<1$ for all $k \in \mathbb{N} \cup\{0\}$.

\section{The Two-Dimensional Lid-Driven Cavity Flow}

In this work we presented the recirculation viscous flow problem in a square cavity, that is called Burggraf Flow [10] [39] [40] [41] [42] [43], and has exact solutions in a steady state as a form

$$
\begin{aligned}
u(x, y)= & 8 f(x) g^{\prime}(y), \\
v(x, y)= & -8 f^{\prime}(x) g(y), \\
p(x, y)= & \frac{8}{\operatorname{Re}}\left(F(x) g^{\prime \prime \prime}(x)+f^{\prime}(x) g^{\prime}(x)\right) \\
& +64 F_{1}(x)\left(g(y) g^{\prime \prime}(y)-\left(g^{\prime}(y)\right)^{2}\right),
\end{aligned}
$$

where

$$
\begin{gathered}
f(x)=x^{4}-2 x^{3}+x^{2}, \quad g(x)=y^{4}-y^{2}, \\
F(x)=\int f(x) \mathrm{d} x, \quad F_{1}(x)=\int f(x) f^{\prime}(x) \mathrm{d} x,
\end{gathered}
$$

such that the stream function $\psi$ and vorticity $\omega$ are defined as

$$
\begin{gathered}
\psi=8 f(x) g(y), \quad \text { such that } \psi_{y}=u, \& \psi_{x}=-v, \\
\omega=v_{x}-u_{y}=-8\left(f^{\prime \prime}(x) g(y)+f(x) g^{\prime \prime}(y)\right) .
\end{gathered}
$$

The boundary conditions for the velocities $u$ and $v$ in this problem are of Dirichlet type, which are equal to zero everywhere except along the top surface where

$$
u(x, 1, t)=16\left(x^{4}-2 x^{3}+x^{2}\right) .
$$

To obtain the approximate analytical solutions of the unsteady lid-driven cavity flow problem, we consider the analytical solutions to this problem, which are given in (5.1) as initial conditions for $u, v$ and $p$.

Then, by applying KRDTM with the initial conditions of this problem, we obtained the iterative solutions like the form (2.5), such that

$$
U_{1}(x, y)=0,
$$




$$
\begin{aligned}
& U_{2}(x, y)=-\frac{192}{(R e)^{2}}\left(12 x^{2}+2 y^{2}-12 x+1\right) y+\frac{256}{5(R e)^{2}}\left[3(2 x-9)\left(6 y^{2}-1\right) x^{8}\right. \\
& -30 y^{4}\left(y^{2}-1\right)^{2}+4\left(531 y^{4}-102 y^{2}+1\right) x^{7}-14\left(531 y^{4}\right. \\
& \left.-156 y^{2}+10\right) x^{6}+\left(2520 y^{6}+7344 y^{4}-2244 y^{2}+215\right) x^{5} \\
& -25\left(252 y^{6}-9 y^{4}+9 y^{2}+5\right) x^{4}+5\left(72 y^{8}+932 y^{6}-660 y^{4}\right. \\
& \left.+264 y^{2}+5\right) x^{3}-5\left(108 y^{6}+138 y^{4}-201 y^{2}+111\right) x^{2} y^{2} \\
& \left.+5\left(48 y^{6}-62 y^{4}+19 y^{2}+11\right) x y^{2}\right] \\
& -1024 x^{2}(x-1)^{2} y\left[6\left(2 y^{6}-5 y^{4}+4 y^{2}-1\right) y^{4}\right. \\
& -44\left(2 y^{2}-1\right) y^{4}\left(y^{2}-1\right)^{2} x+\left(36 y^{6}-18 y^{4}+8 y^{2}-1\right)(x-4) x^{7} \\
& -2\left(90 y^{8}-236 y^{6}+105 y^{4}-37 y^{2}+3\right) x^{6} \\
& +2\left(270 y^{8}-456 y^{6}+189 y^{4}-55 y^{2}+2\right) x^{5} \\
& +\left(144 y^{10}-954 y^{8}+1172 y^{6}-429 y^{4}+93 y^{2}-1\right) x^{4} \\
& -8\left(36 y^{8}-126 y^{6}+124 y^{4}-39 y^{2}+5\right) x^{3} y^{2} \\
& \left.+\left(232 y^{8}-634 y^{6}+544 y^{4}-149 y^{2}+7\right) x^{2} y^{2}\right] \text {, } \\
& V_{1}(x, y)=\frac{32}{R e}\left[3\left(y^{4}-y^{2}+x^{4}-\frac{2}{5} x^{5}\right)-2\left(12 y^{2}-1\right) x^{3}\right. \\
& \left.+6\left(6 y^{2}-1\right) x^{2}-2\left(3 y^{4}+3 y^{2}-1\right) x\right] \\
& +128 x^{2}(x-1)^{2}\left[\left(6 y^{4}-2 y^{2}+1\right)(x-2) x^{3}\right. \\
& \left.-\left(8 y^{6}-18 y^{4}+6 y^{2}-1\right) x^{2}+2\left(2 y^{4}-3 y^{2}+1\right)(2 x-1) y^{2}\right] \text {, } \\
& V_{2}(x, y)=-\frac{192}{(R e)^{2}}\left(12(2 x-1) y^{2}+10 x^{3}-15 x^{2}+x+2\right) \\
& +\frac{256}{5 R e}\left[10 y^{7}+15\left(108 x^{6}-324 x^{5}+334 x^{4}-128 x^{3}+10 x^{2}-1\right) y^{5}\right. \\
& +\left(864 x^{8}-3456 x^{7}+4652 x^{6}-1860 x^{5}-710 x^{4}+480 x^{3}+30 x^{2}+5\right) y^{3} \\
& \left.-x^{2}(x-1) y\left(132 x^{5}-396 x^{4}+130 x^{3}+400 x^{2}-315 x+45\right)\right] \\
& -2048 x^{3}(x-1)^{3}(2 x-1)\left[4\left(16 x^{2}-16 x+9\right) y^{10}\right. \\
& -2\left(15 x^{4}-30 x^{3}+81 x^{2}-66 x+37\right) y^{8} \\
& +2\left(18 x^{2}-18 x+23\right)\left(x^{2}-x+1\right) y^{6} \\
& \left.-\left(7 x^{4}-14 x^{3}+21 x^{2}-14 x+8\right) y^{4}+x^{2}(x-1)^{2} y^{2}\right] \text {, } \\
& G_{1}(x, y)=\frac{192}{(R e)^{2}}\left(4 x^{2}+2 y^{2}-4 x-1\right)(2 x-1) y
\end{aligned}
$$




$$
\begin{gathered}
+\frac{128}{R e}\left[-3\left(50 y^{4}-18 y^{2}+1\right)(x-4) x^{7}\right. \\
-6\left(84 y^{6}+68 y^{4}-32 y^{2}+3\right) x^{6} \\
+12\left(126 y^{6}-73 y^{4}+15 y^{2}+1\right) x^{5} \\
-\left(120 y^{8}+1388 y^{6}-1320 y^{4}+372 y^{2}+3\right) x^{4} \\
+16\left(15 y^{6}+16 y^{4}-30 y^{2}+12\right) x^{3} y^{2} \\
-2\left(78 y^{6}-98 y^{4}+21 y^{2}+15\right) x^{2} y^{2} \\
+2 y^{4}\left(y^{2}-1\right)^{2}(18 x-1) \\
G_{2}(x, y)=\frac{3456}{(R e)^{3} y(2 x-1)+\frac{256}{(R e)^{2}}\left[-9\left(50 y^{2}-3\right)(x-4) x^{7}\right.} \\
-6\left(980 y^{4}+78 y^{2}-9\right) x^{6}+18\left(980 y^{4}-272 y^{2}+12\right) x^{5} \\
-3\left(1820 y^{6}+4490 y^{4}-1800 y^{2}+107\right) x^{4} \\
+12\left(910 y^{6}-205 y^{4}-45 y^{2}+13\right) x^{3} \\
-6\left(60 y^{8}+1058 y^{6}-905 y^{4}+207 y^{2}+4\right) x^{2} \\
+6\left(60 y^{6}+148 y^{4}-210 y^{2}+66\right) y^{2} x-21 y^{2}+9 y^{4} \\
\left.+70 y^{6}-78 y^{8}\right]+\frac{96}{(M a)^{2}}\left(4 e^{2}+2 y^{2}-4 x-1\right) y(2 x-1) \\
+\frac{64}{(M a)^{2}} x^{2}(x-1)^{2}\left[\left(30 y^{4}-6 y^{2}+1\right)(x-2) x^{3}\right. \\
-\left(56 y^{6}-90 y^{4}+18 y^{2}-1\right) x^{2} \\
\left.+2\left(28 y^{6}-30 y^{4}+6 y^{2}\right) x-2\left(14 y^{4}-15 y^{2}+3\right) y^{2}\right] \\
\end{gathered}
$$

To make a decision on the convergence of the KRDTM, we computed $\gamma_{k}$ as:

$$
\begin{gathered}
\gamma_{10}=\frac{\left\|U_{1}(x, y) \tau\right\|}{\left\|U_{0}(x, y)\right\|}=0, \\
\gamma_{20}=\frac{\left\|V_{1}(x, y) \tau\right\|}{\left\|V_{0}(x, y)\right\|}=\frac{\sqrt{714\left(3227504(R e)^{2}-12514788 R e+1994117697\right)}}{14586} \frac{\tau}{R e}, \\
\gamma_{30}=\frac{\left\|G_{1}(x, y) \tau\right\|}{\left\|G_{0}(x, y)\right\|}=\sqrt{\frac{60\left(49405942(R e)^{2}+1467358893\right)}{1253680(R e)^{2}-21900879 R e+292485765}} \frac{\tau}{R e}, \\
\gamma_{11}=\frac{\left\|U_{2}(x, y) \tau^{2}\right\|}{\left\|U_{1}(x, y) \tau\right\|}=0, \\
\frac{\left\|V_{2}(x, y) \tau^{2}\right\|}{\left\|V_{1}(x, y) \tau\right\|}=\tau\left[1 6 4 2 2 \left(4861754265600(R e)^{4}\right.\right. \\
+2305887904320(R e)^{3}+15827448430362608(R e)^{2}
\end{gathered}
$$




$$
\begin{aligned}
& -25967180162523600 R e+3689889931130027625)]^{0.5} \\
& \div\left[260015\left(3227504(R e)^{2}-12514788 R e+1994117697\right)^{0.5} R e\right] \\
\gamma_{31}= & \frac{\left\|G_{2}(x, y) \tau^{2}\right\|}{\left\|G_{1}(x, y) \tau\right\|} \\
= & \tau\left[3 \left(\left(2007409369056(R e)^{2}+14901358071600\right)(M a)^{4}\right.\right. \\
& +\left(1477832512(R e)^{2}-165570645240\right)(R e)^{2}(M a)^{2} \\
& \left.\left.+\left(20792594(R e)^{2}+4402076679\right) R e^{4}\right)\right]^{0.5} \\
& \div\left[6\left(49405942(R e)^{2}+1467358893\right)^{0.5} \operatorname{Re}(M a)^{2}\right]
\end{aligned}
$$

such that $\gamma_{0}=\gamma_{10}+\gamma_{20}+\gamma_{30}, \gamma_{1}=\gamma_{11}+\gamma_{21}+\gamma_{31}, \cdots$. For example, if $M a=0.1$, $t=0.1$, and $R e=1$ such that $\tau=M a \times t$, for all $x$ and $y$ in domain $[0,1]^{2}$, then

$$
\gamma_{0}=0.9991283888<1, \gamma_{1}=0.7958329986<1, \cdots,
$$

if $M a=0.1, t=0.01$, and $R e=10$ then

$$
\gamma_{0}=0.0129736262<1, \gamma_{1}=0.2936820858<1, \cdots
$$

Thus, the iterative solutions (3.2) for this problem, which are obtained by using KPIA, have the following form

$$
\begin{aligned}
& u_{1}(x, y, \tau)=16 x^{2} y\left(2 y^{2}-1\right)(x-1)^{2}, \\
u_{2}(x, y, \tau)= & 16 x^{2} y\left(2 y^{2}-1\right)(x-1)^{2}+\left[-\frac{192 y}{(R e)^{2}}\left(12 x^{2}-12 x+2 y^{2}+1\right)\right. \\
+ & \frac{256}{5 R e}\left(3\left(6 y^{2}-1\right)(2 x-9) x^{8}+4\left(531 y^{4}-102 y^{2}+1\right) x^{7}\right. \\
& -14\left(531 y^{4}-156 y^{2}+10\right) x^{6}+\left(2520 y^{6}+7344 y^{4}\right. \\
& \left.-2244 y^{2}+215\right) x^{5}-25\left(252 y^{6}-9 y^{4}+9 y^{2}+5\right) x^{4} \\
+ & 5\left(72 y^{8}+932 y^{6}-660 y^{4}+264 y^{2}+5\right) x^{3} \\
& -5\left(108 y^{6}+138 y^{4}-201 y^{2}+111\right) x^{2} y^{2} \\
+ & \left.5\left(48 y^{6}-62 y^{4}+19 y^{2}+11\right) x y^{2}-30 y^{4}\left(y^{2}-1\right)^{2}\right) \\
& -1024 x^{2}(x-1)^{2} y\left(\left(36 y^{6}-18 y^{4}+8 y^{2}-1\right)(x-4) x^{7}\right. \\
& -2\left(90 y^{8}-236 y^{6}+105 y^{4}-37 y^{2}+3\right) x^{6} \\
& +2\left(270 y^{8}-456 y^{6}+189 y^{4}-55 y^{2}+2\right) x^{5} \\
+ & \left(144 y^{10}-954 y^{8}+1172 y^{6}-429 y^{4}+93 y^{2}-1\right) x^{4} \\
& -4\left(72 y^{8}-252 y^{6}+248 y^{4}-78 y^{2}+10\right) x^{3} y^{2}
\end{aligned}
$$




$$
\begin{aligned}
& +\left(232 y^{8}-634 y^{6}+544 y^{4}-149 y^{2}+7\right) x^{2} y^{2} \\
& \left.\left.-44\left(2 y^{2}-1\right) y^{4}\left(y^{2}-1\right)^{2} x+6\left(2 y^{6}-5 y^{4}+4 y^{2}-1\right) y^{4}\right)\right] \tau^{2} \\
& -16384\left[\left(-\frac{1}{R e}\left(3(x-2) x^{3}+3\left(12 y^{2}-1\right) x^{2}-6\left(6 y^{2}-1\right) x\right.\right.\right. \\
& \left.+3 y^{4}+3 y^{2}-1\right)+8 y\left(\left(6 y^{4}-2 y^{2}+1\right)(2 x-7) x^{6}\right. \\
& -3\left(4 y^{6}-24 y^{4}+8 y^{2}-3\right) x^{5}+5\left(6 y^{6}-15 y^{4}+5 y^{2}-1\right) x^{4} \\
& \left.\left.-\left(28 y^{6}-48 y^{4}+16 y^{2}-1\right) x^{3}+\left(2 y^{4}-3 y^{2}+1\right)(6 x-1) x y^{2}\right)\right) \\
& \times\left(-\frac{1}{120 \operatorname{Re}}\left(3(2 x-5) x^{4}+10\left(12 y^{2}-1\right) x^{3}\right.\right. \\
& \left.-30\left(6 y^{2}-1\right) x^{2}+10\left(3 y^{4}+3 y^{2}-1\right) x-15 y^{2}\left(y^{2}-1\right)\right) \\
& +\frac{y}{6}\left(\left(6 y^{4}-2 y^{2}+1\right)(x-4) x^{7}-2\left(4 y^{6}-24 y^{4}+8 y^{2}-3\right) x^{6}\right. \\
& +4\left(6 y^{6}-15 y^{4}+5 y^{2}-1\right) x^{5}-\left(28 y^{6}-48 y^{4}+16 y^{2}-1\right) x^{4} \\
& \left.\left.\left.+2\left(2 y^{4}-3 y^{2}+1\right)(4 x-1) x^{2} y^{2}\right)\right)\right] \tau^{3}, \\
& v_{1}(x, y, \tau)=-16\left(2 x^{3}-3 x^{2}+x\right)\left(y^{4}-y^{2}\right)+\left[\frac { 3 2 } { \operatorname { R e } } \left(3\left(y^{4}-y^{2}+x^{4}-\frac{2 x^{5}}{5}\right)\right.\right. \\
& \left.-2\left(12 y^{2}-1\right) x^{3}+6\left(6 y^{2}-1\right) x^{2}-2\left(3 y^{4}+3 y^{2}-1\right) x\right) \\
& +128 x^{2}(x-1)^{2} y\left(\left(6 y^{4}-2 y^{2}+1\right)(x-2) x^{3}\right. \\
& \left.\left.-\left(8 y^{6}-18 y^{4}+6 y^{2}-1\right) x^{2}+2\left(2 y^{4}-3 y^{2}+1\right) y^{2}(2 x-1)\right)\right] \tau, \\
& v_{2}(x, y, \tau)=-16\left(2 x^{3}-3 x^{2}+x\right)\left(y^{4}-y^{2}\right)+128\left[y \left(\left(6 y^{4}-2 y^{2}+1\right)(x-4) x^{7}\right.\right. \\
& -2\left(4 y^{6}-24 y^{4}+8 y^{2}-3\right) x^{6}+2\left(12 y^{6}-30 y^{4}+10 y^{2}-2\right) x^{5} \\
& \left.-\left(28 y^{6}-48 y^{4}+16 y^{2}-1\right) x^{4}+2\left(2 y^{4}-3 y^{2}+1\right)(4 x-1) x^{2} y^{2}\right) \\
& -\frac{1}{20 \operatorname{Re}}\left(3(2 x-5) x^{4}+10\left(12 y^{2}-1\right) x^{3}-30\left(6 y^{2}-1\right) x^{2}\right. \\
& \left.\left.+10\left(3 y^{4}+3 y^{2}-1\right) x-15 y^{2}\left(y^{2}-1\right)\right)\right] \tau+\left[-\frac{192}{(\operatorname{Re})^{2}}(2 x-1)\right. \\
& \times\left(5 x^{2}-5 x+12 y^{2}-2\right)+\frac{256 y}{5 R e}\left(12\left(72 y^{2}-11\right)(x-4) x^{7}\right. \\
& +2\left(810 y^{4}+2326 y^{2}-263\right) x^{6}-30\left(162 y^{4}+62 y^{2}+9\right) x^{5} \\
& +5\left(1002 y^{4}-142 y^{2}+143\right) x^{4}-120\left(16 y^{4}-4 y^{2}+3\right) x^{3} \\
& \left.+15\left(10 y^{4}+2 y^{2}+3\right) x^{2}+5\left(2 y^{4}-3 y^{2}+1\right) y^{2}\right) \\
& +512\left(y^{2}-1\right)(x-1)^{3} x^{3}(2 x-1) y^{2}\left(x^{2}(x-1)^{2}\right.
\end{aligned}
$$




$$
\begin{aligned}
& -4\left(16 x^{2}-16 x+9\right) y^{6}+2\left(15 x^{2}-15 x+19\right)\left(x^{2}-x+1\right) y^{4} \\
& \left.\left.-2\left(3 x^{2}-3 x+4\right)\left(x^{2}-x+1\right) y^{2}\right)\right] \tau^{2}+\left[-\frac{512}{15}\left(2 0 y \left(\left(6 y^{4}\right.\right.\right.\right. \\
& \left.-2 y^{2}+1\right)(x-4) x^{7}-2\left(4 y^{6}-24 y^{4}+8 y^{2}-3\right) x^{6} \\
& +2\left(12 y^{6}-30 y^{4}+10 y^{2}-2\right) x^{5}-\left(28 y^{6}-48 y^{4}+16 y^{2}-1\right) x^{4} \\
& \left.+2\left(2 y^{4}-3 y^{2}+1\right)(4 x-1) x^{2} y^{2}\right)-\frac{1}{R e}\left(3(2 x-5) x^{4}\right. \\
& +10\left(12 y^{2}-1\right) x^{3}-30\left(6 y^{2}-1\right) x^{2}+10\left(3 y^{4}+3 y^{2}-1\right) x \\
& \left.\left.-15 y^{2}\left(y^{2}-1\right)\right)\right) \times\left(2\left(30 y^{4}-6 y^{2}+1\right)(x-4) x^{7}\right. \\
& -4\left(28 y^{6}-120 y^{4}+24 y^{2}-3\right) x^{6}+8\left(42 y^{6}-75 y^{4}\right. \\
& \left.+15 y^{2}-1\right) x^{5}-2\left(196 y^{6}-240 y^{4}+48 y^{2}-1\right) x^{4} \\
& +16\left(14 y^{6}-15 y^{4}+3 y^{2}\right) x^{3}-4\left(14 y^{6}-15 y^{4}+3 y^{2}\right) x^{2} \\
& \left.\left.-\frac{3 y}{R e}\left(4(2 x-3) x^{2}+2\left(2 y^{2}+1\right)\right) x-2 y^{2}+1\right)\right] \tau^{3}, \\
& g_{1}(x, y, \tau)=\frac{32 x y}{\operatorname{Re}}\left[\left(4 y^{2}-3 x+\frac{6 x^{2}}{5}\right) x^{2}-\left(2 y^{2}-1\right)(3 x-1)\right] \\
& -64(x-1)^{2} x^{2} y^{2}\left[\left(10 y^{4}-9 y^{2}+3\right)(x-2) x^{3}\right. \\
& \left.+\left(8 y^{6}-6 y^{4}-y^{2}+3\right) x^{2}-2 y^{2}\left(y^{2}-1\right)^{2}(4 x-1)\right] \\
& -\left[\frac{192}{(\operatorname{Re})^{2}}(2 x-1)\left(4 x^{2}+2 y^{2}-4 x-1\right) y\right. \\
& -\frac{128}{R e}\left(3\left(50 y^{4}-18 y^{2}+1\right)(x-4) x^{7}+6\left(84 y^{6}+68 y^{4}\right.\right. \\
& \left.-32 y^{2}+3\right) x^{6}-6\left(252 y^{6}-146 y^{4}+30 y^{2}+2\right) x^{5} \\
& +\left(120 y^{8}+1388 y^{6}-1320 y^{4}+372 y^{2}+3\right) x^{4} \\
& -16\left(15 y^{6}+16 y^{4}-30 y^{2}+12\right) x^{3} y^{2}+2\left(78 y^{6}-98 y^{4}\right. \\
& \left.\left.\left.+21 y^{2}+15\right) x^{2} y^{2}-2(18 x-1) y^{4}\left(y^{2}-1\right)^{2}\right)\right] \tau, \\
& g_{2}(x, y, \tau)=-64 x^{2}(x-1)^{2} y^{2}\left(\left(10 y^{4}-9 y^{2}+3\right)(x-2) x^{3}\right. \\
& \left.+\left(8 y^{6}-6 y^{4}-y^{2}+3\right) x^{2}-2\left(y^{2}-1\right)^{2}(4 x-1) y^{2}\right) \\
& +\frac{32 y}{5 \operatorname{Re}}\left(3(2 x-5) x^{4}+20 x^{3} y^{2}-5\left(2 y^{2}-1\right)(3 x-1) x\right) \\
& +\left[\frac{192 y}{(R e)^{2}}(2 x-1)\left(4 x^{2}-4 x+2 y^{2}-1\right)-\frac{128}{R e}\left(3 \left(50 y^{4}\right.\right.\right. \\
& \left.-18 y^{2}+1\right)(x-4) x^{7}+6\left(84 y^{6}+68 y^{4}-32 y^{2}+3\right) x^{6} \\
& -12\left(126 y^{6}-73 y^{4}+15 y^{2}+1\right) x^{5}+\left(120 y^{8}+1388 y^{6}\right.
\end{aligned}
$$




$$
\begin{aligned}
& \left.-1320 y^{4}+372 y^{2}+3\right) x^{4}-16\left(15 y^{6}+16 y^{4}-30 y^{2}+12\right) x^{3} y^{2} \\
& \left.\left.+2\left(78 y^{6}-98 y^{4}+21 y^{2}+15\right) x^{2} y^{2}-2 y^{4}\left(y^{2}-1\right)^{2}(18 x-1)\right)\right] \tau \\
& +\left[\frac{3456 y}{(R e)^{3}}(2 x-1)-\frac{256}{(R e)^{2}}\left(9\left(50 y^{2}-3\right)(x-4) x^{7}\right.\right. \\
& +6\left(980 y^{4}+78 y^{2}-9\right) x^{6}-72\left(245 y^{4}-68 y^{2}+3\right) x^{5} \\
& +3\left(1820 y^{6}+4490 y^{4}-1800 y^{2}+107\right) x^{4}-12\left(910 y^{6}\right. \\
& \left.-205 y^{4}-45 y^{2}+13\right) x^{3}+2\left(180 y^{8}+3174 y^{6}-2715 y^{4}\right. \\
& \left.+621 y^{2}+12\right) x^{2}-12\left(30 y^{6}+74 y^{4}-105 y^{2}+33\right) x y^{2} \\
& \left.+\left(78 y^{6}-70 y^{4}-9 y^{2}+21\right) y^{2}\right)+\frac{1}{(M a)^{2}}\left(\frac { 9 6 y } { R e } \left(4(2 x-3) x^{2}\right.\right. \\
& \left.+2\left(2 y^{2}+1\right) x-2 y^{2}+1\right)-64 x^{2}(x-1)^{2}\left(\left(30 y^{4}-6 y^{2}+1\right)(x-2) x^{3}\right. \\
& \left.\left.\left.-\left(56 y^{6}-90 y^{4}+18 y^{2}-1\right) x^{2}+2\left(14 y^{4}-15 y^{2}+3\right)(2 x-1) y^{2}\right)\right)\right] \tau^{2},
\end{aligned}
$$

To test the convergence of the approximate solutions, we calculated $\gamma_{k}$ as:

$$
\begin{aligned}
& \gamma_{10}=\frac{\left\|\left(u_{c}\right)_{1}(x, y, \tau)\right\|}{\|u(x, y, 0)\|}=0, \quad \gamma_{11}=\frac{\left\|\left(u_{c}\right)_{2}(x, y, \tau)\right\|}{\left\|\left(u_{c}\right)_{1}(x, y, \tau)\right\|}=0, \\
& \gamma_{20}=\frac{\left\|\left(v_{c}\right)_{1}(x, y, \tau)\right\|}{\|v(x, y, 0)\|} \\
& =\frac{\sqrt{714\left(3227504(R e)^{2}-12514788 R e+1994117697\right)}}{14586} \frac{\tau}{R e}, \\
& \gamma_{30}=\frac{\left\|\left(g_{c}\right)_{1}(x, y, \tau)\right\|}{\|g(x, y, 0)\|}=\sqrt{\frac{60\left(49405942(R e)^{2}+1467358893\right)}{1253680(R e)^{2}-21900879 R e+292485765}} \frac{\tau}{R e}, \\
& \gamma_{21}=\frac{\left\|\left(v_{c}\right)_{2}(x, y, \tau)\right\|}{\left\|\left(v_{c}\right)_{1}(x, y, \tau)\right\|}=2 \sqrt{534905} \tau\left[\left(252795448503308124160 \tau^{2}\right.\right. \\
& +13571076548224512000)(R e)^{4} \\
& -1150099707715306782720\left(\tau^{2}+\frac{20037330051}{41150401552} \tau-\frac{369495}{66021376}\right)(R e)^{3} \\
& +\left(71129205032874519404544 \tau^{2}+4131885499121329297920 \tau\right. \\
& +44180660411272032158160)(R e)^{2} \\
& -185898066637793079398400\left(\tau^{2}+\frac{19609947}{23628440} \tau+\frac{5360355}{13747456}\right) R e \\
& \left.+7961346208200723126497280\left(\tau^{2}+\frac{34459425}{275978504} \tau+\frac{182807249625}{141300994048}\right)\right]^{0.5} \\
& \div\left[3506302275\left(3227504(R e)^{2}-12514788 R e+1994117697\right)^{0.5} R e\right] \text {, }
\end{aligned}
$$




$$
\begin{aligned}
\gamma_{31}= & \frac{\left\|\left(g_{c}\right)_{2}(x, y, \tau)\right\|}{\left\|\left(g_{c}\right)_{1}(x, y, \tau)\right\|} \\
= & \frac{\tau}{\operatorname{Re}(M a)^{2}}\left[\left(\left(2007409369056(R e)^{2}+14901358071600\right)(M a)^{4}\right.\right. \\
& +\left(1477832512(R e)^{2}-165570645240\right)(\operatorname{Re})^{2}(M a)^{2} \\
& \left.+\left(20792594(R e)^{2}+4402076679\right)(R e)^{4}\right) \\
& \left.\div\left(12\left(49405942(R e)^{2}+1467358893\right)\right)\right]^{0.5}
\end{aligned}
$$

such that $\gamma_{0}=\gamma_{10}+\gamma_{20}+\gamma_{30}, \gamma_{1}=\gamma_{11}+\gamma_{21}+\gamma_{31}, \cdots$. For example, if $M a=0.1$, $t=0.1$, and $R e=1$ such that $\tau=M a \times t$, then

$$
\gamma_{0}=0.9991283888<1, \gamma_{1}=0.7959676332<1, \cdots,
$$

if $M a=0.1, t=0.01$, and $R e=10$ then

$$
\gamma_{0}=0.0129736262<1, \gamma_{1}=0.2936820051<1, \cdots
$$

\section{Results and Discussion}

In this section, we introduce the numerical computations of velocity components $u$, $V$, vorticity function $w$ and stream function $\psi$, which have been obtained by the application of KRDTM and KPIA. All calculations are run by Maple 2017 software with used various values of Reynolds numbers and Mach numbers in the domain $[0,1]^{2}$.

In Table 2 and Table 3, we reviewed the calculated values of $u$ velocity along the vertical line and $v$ velocity along the horizontal line through the geometric center of the square cavity by using KRDTM and KPIA at $t=0.1$ and $M a=0.01$ for different Reynolds numbers. By comparing the results of these methods, we observe they are close to each other for the different values of Reynolds numbers. In Table 4, we compare the results obtained from these

Table 2. The approximate solutions by KRDTM for $u$ and $v$ at $t=0.1$.

\begin{tabular}{ccccc}
\hline$u(0.5, y, 0.1)$ & $R e=10$ & $R e=100$ & $R e=400$ & $R e=1000$ \\
\hline 0.0625 & -0.0620116263 & -0.0620117178 & -0.0620117080 & -0.0620117057 \\
0.125 & -0.1210934186 & -0.1210937382 & -0.1210937303 & -0.1210937281 \\
0.1875 & -0.1743158597 & -0.1743163948 & -0.1743163886 & -0.1743163864 \\
0.25 & -0.2187492697 & -0.2187500032 & -0.2187499985 & -0.2187499963 \\
0.3125 & -0.2514639638 & -0.2514648747 & -0.2514648714 & -0.2514648693 \\
0.375 & -0.2695302503 & -0.2695313138 & -0.2695313119 & -0.2695313098 \\
0.4375 & -0.2700184296 & -0.2700196175 & -0.2700196172 & -0.2700196153 \\
0.5 & -0.2499987981 & -0.2500000797 & -0.2500000812 & -0.2500000796 \\
0.5625 & -0.2065416607 & -0.2065430027 & -0.2065430066 & -0.2065430054 \\
0.625 & -0.1367173508 & -0.1367187180 & -0.1367187250 & -0.1367187244 \\
0.6875 & -0.0375962574 & -0.0375976129 & -0.0375976240 & -0.0375976244 \\
\hline
\end{tabular}




\section{Continued}

\begin{tabular}{|c|c|c|c|c|}
\hline 0.75 & 0.0937511524 & 0.0937498465 & 0.0937498298 & 0.0937498282 \\
\hline 0.8125 & 0.2602543373 & 0.2602531196 & 0.2602530957 & 0.2602530924 \\
\hline 0.875 & 0.4648427531 & 0.4648416627 & 0.4648416294 & 0.4648416238 \\
\hline 0.9375 & 0.7104460577 & $\begin{array}{l}0.7104451334 \\
v(x, 0.5,0.1)\end{array}$ & 0.7104450882 & 0.7104450798 \\
\hline 0.0625 & 0.1520352246 & 0.1535990206 & 0.1537294237 & 0.1537555060 \\
\hline 0.125 & 0.2443698776 & 0.2458276287 & 0.2459491556 & 0.2459734619 \\
\hline 0.1875 & 0.2840233785 & 0.2853353452 & 0.2854446967 & 0.2854665674 \\
\hline 0.25 & 0.2797924904 & 0.2809293365 & 0.2810240781 & 0.2810430265 \\
\hline 0.3125 & 0.2404575825 & 0.2413982264 & 0.2414766095 & 0.2414922860 \\
\hline 0.375 & 0.1747890513 & 0.1755190079 & 0.1755798316 & 0.1755919962 \\
\hline 0.4375 & 0.0915546641 & 0.0920648498 & 0.0921073612 & 0.0921158634 \\
\hline 0.5 & -0.0004740000 & -0.0001880250 & -0.0001641937 & -0.0001594275 \\
\hline 0.5625 & -0.0925173262 & -0.0924556955 & -0.0924505555 & -0.0924495275 \\
\hline 0.625 & -0.1757864500 & -0.1759449258 & -0.1759581261 & -0.1759607661 \\
\hline 0.6875 & -0.2414853287 & -0.2418548488 & -0.2418856383 & -0.2418917961 \\
\hline 0.75 & -0.2808158432 & -0.2813816962 & -0.2814288551 & -0.2814382869 \\
\hline 0.8125 & -0.2849851434 & -0.2857257912 & -0.2857875328 & -0.2857998815 \\
\hline 0.875 & -0.2452139088 & -0.2460994800 & -0.2461733253 & -0.2461880953 \\
\hline 0.9375 & -0.1527436594 & -0.1537341432 & -0.1538167704 & -0.1538332975 \\
\hline
\end{tabular}

Table 3. The approximate solution by KPIA for $u$ and $v$ at $t=0.1$.

\begin{tabular}{|c|c|c|c|c|}
\hline$u(0.5, y, 0.1)$ & $\operatorname{Re}=10$ & $R e=100$ & $R e=400$ & $\operatorname{Re}=1000$ \\
\hline 0.0625 & -0.0620116267 & -0.0620117178 & -0.0620117080 & -0.0620117057 \\
\hline 0.125 & -0.1210934190 & -0.1210937382 & -0.1210937303 & -0.1210937281 \\
\hline 0.1875 & -0.1743158600 & -0.1743163948 & -0.1743163886 & -0.1743163864 \\
\hline 0.25 & -0.2187492699 & -0.2187500032 & -0.2187499985 & -0.2187499963 \\
\hline 0.3125 & -0.2514639639 & -0.2514648747 & -0.2514648714 & -0.2514648693 \\
\hline 0.375 & -0.2695302503 & -0.2695313137 & -0.2695313119 & -0.2695313098 \\
\hline 0.4375 & -0.2700184292 & -0.2700196175 & -0.2700196172 & -0.2700196153 \\
\hline 0.5 & -0.2499987975 & -0.2500000797 & -0.2500000812 & -0.2500000796 \\
\hline 0.5625 & -0.2065416599 & -0.2065430027 & -0.2065430066 & -0.2065430054 \\
\hline 0.625 & -0.1367173502 & -0.1367187180 & -0.1367187250 & -0.1367187244 \\
\hline 0.6875 & -0.0375962576 & -0.0375976130 & -0.0375976241 & -0.0375976244 \\
\hline 0.75 & 0.0937511505 & 0.0937498462 & 0.0937498298 & 0.0937498282 \\
\hline 0.8125 & 0.2602543328 & 0.2602531191 & 0.2602530956 & 0.2602530923 \\
\hline 0.875 & 0.4648427455 & 0.4648416618 & 0.4648416292 & 0.4648416237 \\
\hline \multirow[t]{2}{*}{0.9375} & 0.7104460475 & 0.7104451322 & 0.7104450879 & 0.7104450797 \\
\hline & & $v(x, 0.5,0.1)$ & & \\
\hline 0.0625 & 0.1520352173 & 0.1535990205 & 0.1537294237 & 0.1537555060 \\
\hline 0.125 & 0.2443698698 & 0.2458276285 & 0.2459491556 & 0.2459734619 \\
\hline 0.1875 & 0.2840233713 & 0.2853353451 & 0.2854446967 & 0.2854665674 \\
\hline
\end{tabular}




\section{Continued}

\begin{tabular}{ccccc}
\hline 0.25 & 0.2797924846 & 0.2809293364 & 0.2810240781 & 0.2810430265 \\
0.3125 & 0.2404575786 & 0.2413982263 & 0.2414766095 & 0.2414922860 \\
0.375 & 0.1747890492 & 0.1755190079 & 0.1755798316 & 0.1755919962 \\
0.4375 & 0.0915546634 & 0.0920648498 & 0.0921073612 & 0.0921158634 \\
0.5 & -0.0004739999 & -0.0001880249 & -0.0001641937 & -0.0001594275 \\
0.5625 & -0.0925173258 & -0.0924556954 & -0.0924505555 & -0.0924495274 \\
0.625 & -0.1757864500 & -0.1759449258 & -0.1759581261 & -0.1759607660 \\
0.6875 & -0.2414853294 & -0.2418548488 & -0.2418856382 & -0.2418917961 \\
0.75 & -0.2808158450 & -0.2813816962 & -0.2857875328 & -0.2814382869 \\
0.8125 & -0.2849851463 & -0.2857257912 & -0.2461733253 & -0.2857998815 \\
0.875 & -0.2452139127 & -0.2460994800 & -0.2461733253 & -0.2461880953 \\
0.9375 & -0.1527436637 & -0.1537341433 & -0.1538167704 & -0.1538332975 \\
\hline
\end{tabular}

Table 4. Comparison between the approximate solutions at $t=0.1$ and $R e=1$.

\begin{tabular}{|c|c|c|c|}
\hline & Ref. [10] & KRDTM & KPIA \\
\hline$\psi_{\min }$ & -0.125 & -0.1263030704 & -0.1262878352 \\
\hline$x\left(\psi_{\min }\right)$ & 0.5 & 0.5 & 0.5 \\
\hline$y\left(\psi_{\min }\right)$ & 0.70703 & 0.70703125 & 0.703125 \\
\hline$u_{\min }$ & -0.2721659 & -0.2720274424 & -0.2720273443 \\
\hline$y\left(u_{\min }\right)$ & 0.40869 & 0.41015625 & 0.40625 \\
\hline$v_{\min }$ & -0.2886756 & -0.365196882 & -0.3649970593 \\
\hline$x\left(v_{\min }\right)$ & 0.78857 & 0.78515625 & 0.78125 \\
\hline$v_{\max }$ & 0.2886756 & 0.3590485063 & 0.3588814265 \\
\hline$x\left(v_{\max }\right)$ & 0.21143 & 0.21484375 & 0.21875 \\
\hline$u(0.5 ; 0.0625)$ & -0.062011718741 & -0.0619894102 & -0.0619894551 \\
\hline$u(0.5 ; 0.125)$ & -0.121093749988 & -0.1210481233 & -0.1210481633 \\
\hline$u(0.5 ; 0.1875)$ & -0.174316406238 & -0.1742486127 & -0.1742486451 \\
\hline$u(0.5 ; 0.25)$ & -0.218749999990 & -0.2186617447 & -0.2186617667 \\
\hline$u(0.5 ; 0.3125)$ & -0.251464843745 & -0.2513583768 & -0.2513583858 \\
\hline$u(0.5 ; 0.375)$ & -0.2695312499997 & -0.2694093550 & -0.2694093482 \\
\hline$u(0.5 ; 0.4375)$ & -0.270019531254 & -0.2698855135 & -0.2698854884 \\
\hline$u(0.5 ; 0.5)$ & -0.250000000006 & -0.2498576781 & -0.2498576334 \\
\hline$u(0.5 ; 0.5625)$ & -0.206542968755 & -0.2063966790 & -0.2063966152 \\
\hline$u(0.5 ; 0.625)$ & -0.1367187500006 & -0.1365733705 & -0.1365732911 \\
\hline$u(0.5 ; 0.6875)$ & -0.037597656248 & -0.0374586584 & -0.0374585703 \\
\hline$u(0.5 ; 0.75)$ & 0.093749999998 & 0.0938764774 & 0.0938765646 \\
\hline$u(0.5 ; 0.8125)$ & 0.260253906243 & 0.2603609875 & 0.2603610630 \\
\hline$u(0.5 ; 0.875)$ & 0.46484374998 & 0.4649238234 & 0.4649238796 \\
\hline$u(0.5 ; 0.9375)$ & 0.710449218737 & 0.7104941432 & 0.7104941808 \\
\hline
\end{tabular}




\section{Continued}

\begin{tabular}{cccc}
\hline$v(0.0625 ; 0.5)$ & 0.153808593744 & 0.1365030994 & 0.1365023819 \\
$v(0.125 ; 0.5)$ & 0.24609374999 & 0.2298505049 & 0.2298497694 \\
$v(0.1875 ; 0.5)$ & 0.285644533123 & 0.2709291888 & 0.2709285329 \\
$v(0.25 ; 0.5)$ & 0.28124999999 & 0.2684293744 & 0.2684288605 \\
$v(0.3125 ; 0.5)$ & 0.241699218747 & 0.2310463824 & 0.2310460353 \\
$v(0.375 ; 0.5)$ & 0.175781250002 & 0.1674821344 & 0.1674819447 \\
$v(0.4375 ; 0.5)$ & 0.092285156254 & 0.0864478792 & 0.0864478112 \\
$v(0.5 ; 0.5)$ & $2.3 \mathrm{e}-14$ & -0.00333375 & -0.003333749 \\
$v(0.5625 ; 0.5)$ & -0.092285156254 & -0.0931287051 & -0.0931286946 \\
$v(0.625 ; 0.5)$ & -0.175781250002 & -0.1741943413 & -0.1741943774 \\
$v(0.6875 ; 0.5)$ & -0.241699218746 & -0.2377853663 & -0.2377854934 \\
$v(0.75 ; 0.5)$ & -0.281249999989 & -0.2751626592 & -0.2751629021 \\
$v(0.8125 ; 0.5)$ & -0.28564453123 & -0.2776041425 & -0.2776044993 \\
$v(0.875 ; 0.5)$ & -0.24609374999 & -0.2364163347 & -0.2364167719 \\
$v(0.9375 ; 0.5)$ & -0.153808593744 & -0.1429446548 & -0.1429451057 \\
\hline
\end{tabular}

Table 5. Comparisons of the $L^{\infty}$-errors at $t=0.1$ and $M a=0.001$.

\begin{tabular}{|c|c|c|c|c|c|c|}
\hline \multirow[t]{2}{*}{ Grid size } & \multicolumn{2}{|l|}{ Ref. [43] } & \multirow{2}{*}{$\begin{array}{c}\text { KRDTM } \\
\psi\end{array}$} & \multicolumn{3}{|c|}{ KPIA } \\
\hline & $\psi$ & $\omega$ & & $\omega$ & $\psi$ & $\omega$ \\
\hline \multicolumn{7}{|c|}{$R e=10$} \\
\hline $21 \times 21$ & $3.230 \mathrm{e}-7$ & $1.008 \mathrm{e}-5$ & $2.0098 \mathrm{e}-8$ & $8.1319 \mathrm{e}-7$ & $5.8382 \mathrm{e}-8$ & $1.5946 \mathrm{e}-6$ \\
\hline $41 \times 41$ & $2.347 \mathrm{e}-8$ & $7.740 \mathrm{e}-7$ & $3.4138 \mathrm{e}-8$ & $9.7680 \mathrm{e}-7$ & $7.2296 \mathrm{e}-8$ & $1.8527 \mathrm{e}-6$ \\
\hline $81 \times 81$ & $1.559 \mathrm{e}-9$ & $5.168 \mathrm{e}-8$ & $4.4644 \mathrm{e}-8$ & $1.0757 e-7$ & $9.2885 \mathrm{e}-8$ & $2.0028 \mathrm{e}-6$ \\
\hline \multicolumn{7}{|c|}{$R e=100$} \\
\hline $21 \times 21$ & $8.087 e-5$ & $4.081 \mathrm{e}-3$ & $4.2498 \mathrm{e}-9$ & $3.6230 \mathrm{e}-7$ & $4.2494 \mathrm{e}-9$ & $3.6230 \mathrm{e}-7$ \\
\hline $41 \times 41$ & $7.120 \mathrm{e}-6$ & $2.508 \mathrm{e}-4$ & $5.1135 \mathrm{e}-9$ & $3.6230 \mathrm{e}-7$ & $5.1130 \mathrm{e}-9$ & $3.6230 \mathrm{e}-7$ \\
\hline $81 \times 81$ & $4.927 \mathrm{e}-7$ & $1.717 e-5$ & $5.6559 \mathrm{e}-9$ & $3.6372 \mathrm{e}-7$ & $5.6555 \mathrm{e}-9$ & $3.6372 \mathrm{e}-7$ \\
\hline \multicolumn{7}{|c|}{$R e=1000$} \\
\hline $41 \times 41$ & $3.322 \mathrm{e}-4$ & $1.449 \mathrm{e}-2$ & $5.0673 \mathrm{e}-9$ & $3.6448 \mathrm{e}-7$ & $5.0669 \mathrm{e}-9$ & $3.6449 \mathrm{e}-7$ \\
\hline $81 \times 81$ & $3.916 \mathrm{e}-5$ & $1.659 \mathrm{e}-3$ & $5.7419 \mathrm{e}-9$ & $3.6448 \mathrm{e}-7$ & $5.7419 \mathrm{e}-9$ & $3.6449 \mathrm{e}-7$ \\
\hline $161 \times 161$ & $2.795 \mathrm{e}-6$ & $1.469 \mathrm{e}-4$ & $6.1146 \mathrm{e}-9$ & $3.6448 \mathrm{e}-7$ & $6.1146 \mathrm{e}-9$ & $3.6449 \mathrm{e}-7$ \\
\hline
\end{tabular}

methods at $R e=1, M a=0.01$ and $t=0.1$ with the numerical results which have been evaluated by using the finite volume method and introduced by [10]. By comparison, we note that our solutions are remarkably good, and these results represent solutions for the second iteration step. The $L^{\infty}$-errors for stream function $\psi$ and vorticity $\omega$ are given in Table 5 for Reynolds numbers $R e=10,100$ and 1000 at $M a=0.001$, are compared with the calculated errors by the rational fourth-order compact finite difference method in [43]. We note 
that the calculated errors are small for all values of the Reynolds number and are not affected by the number of grid points. Also, the approximate solutions of velocity in the two directions at cavity center, which are obtained by KRDTM and KPIA, are shown in Figure 1 at $t=2$ and $R e=10$ for three different values of Mach numbers, and in Figure 2 at $R e=1$ and $M a=0.01$ for three different time levels. We observe that the results of KRDTM and KPIA methods at $M a=0.01$ are better than the results at other Mach numbers. Thus, the numerical results of both methods are good and close to each other at low values of Mach numbers.

\section{Conclusion}

In this paper, we applied the reduced differential transform method and the perturbation-iteration algorithm on the kinetically reduced local Navier-Stokes equations to find approximate solutions to the problem of lid-driven square cavity flow. The calculations in this study show that KRDTM and KPIA are fast and
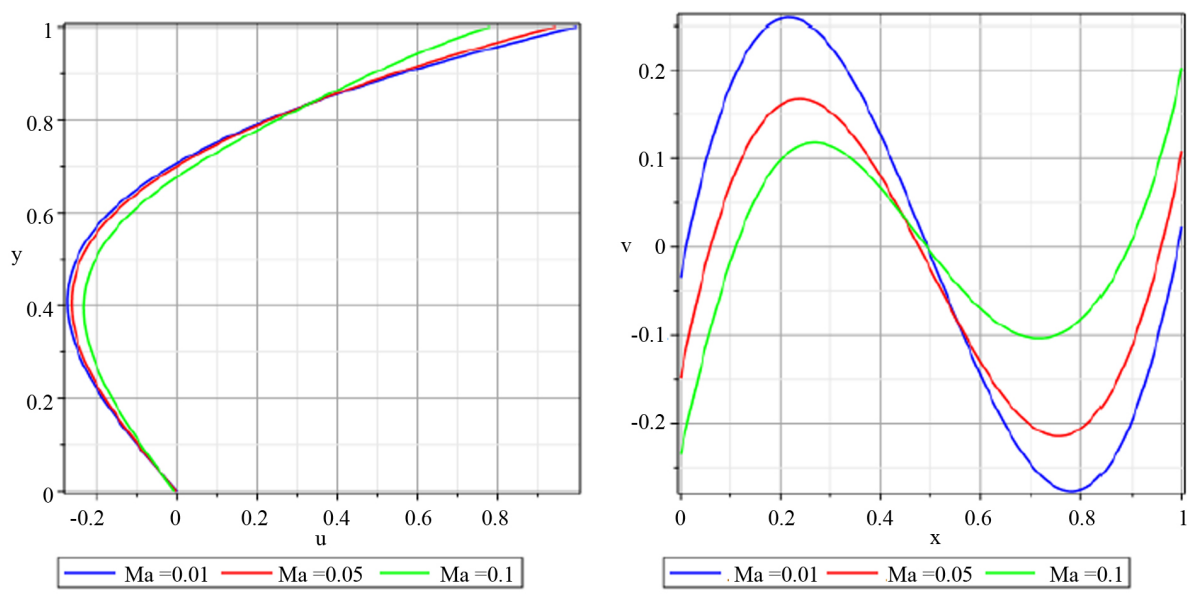

(a)
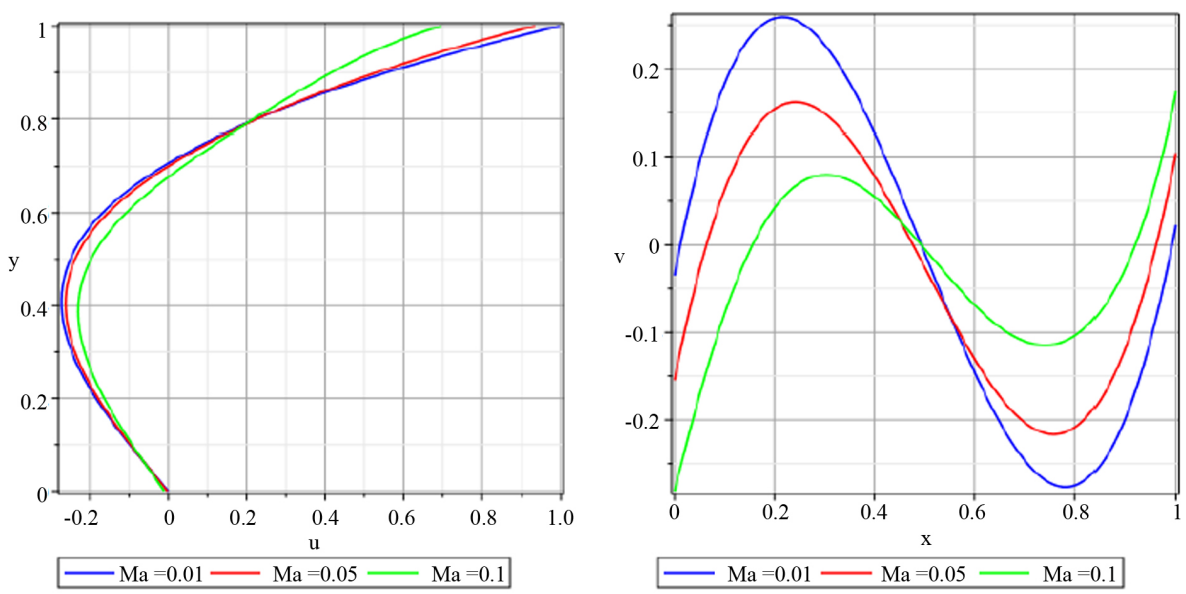

(b)

Figure 1. The approximate solutions of $u(0.5, y, 2)$ and $v(x, 0.5,2) \quad R e=10$. (a) KRDTM; (b) KPIA. 

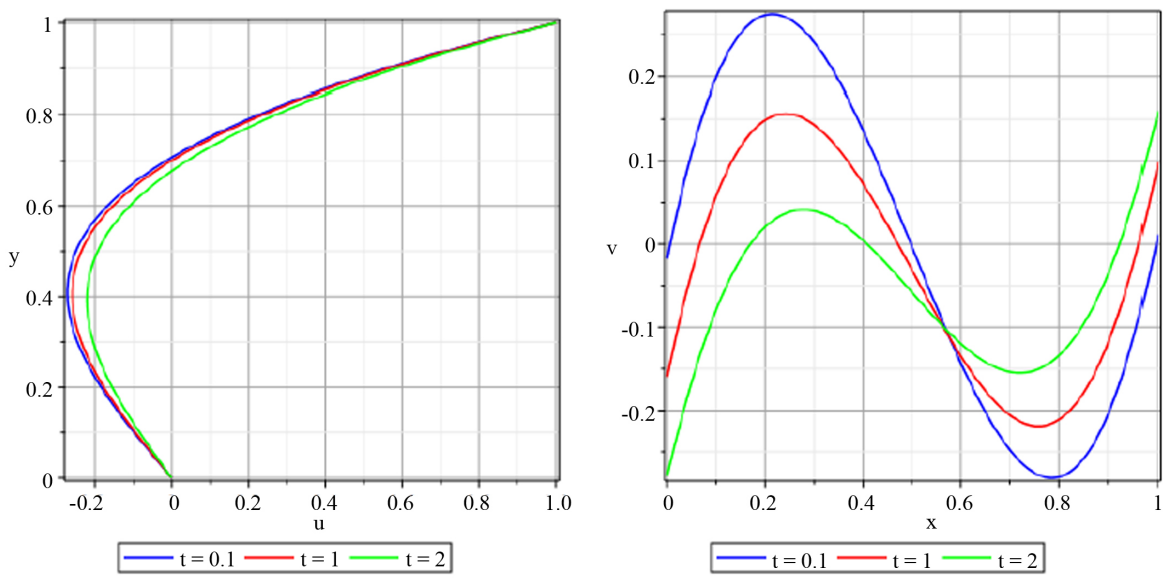

(a)
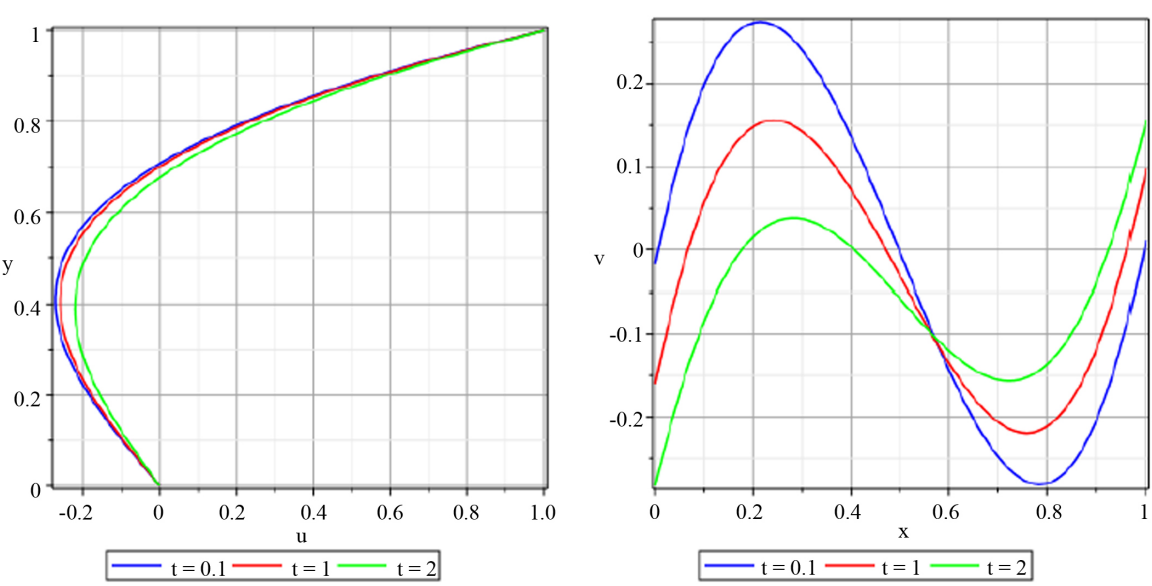

(b)

Figure 2. The approximate solutions of $u(0.5, y, t)$ and $v(x, 0.5, t)$ at $\operatorname{Re}=1$. (a) KRDTM; (b) KPIA.

successful techniques and yield remarkably good results to solve unsteady viscous incompressible flow problems at low Mach numbers. Therefore, the application of KRDTM and KPIA could be expanded to include various and multi-dimensions of flow problems. In addition, these methods can be combined with other methods to increase the accuracy of solutions.

\section{Conflicts of Interest}

The authors declare no conflicts of interest regarding the publication of this paper.

\section{References}

[1] Ansumali, S., Karlin, I.V. and Öttinger, H.C. (2005) Thermodynamic Theory of Incompressible Hydrodynamics. Physical Review Letters, 94, Article ID: 080602. https://doi.org/10.1103/PhysRevLett.94.080602

[2] Borok, S., Ansumali, S. and Karlin, I.V. (2007) Kinetically Reduced Local Navier-Stokes Equations for Simulation of Incompressible Viscous Flows. Physical Re- 
view E, 76, Article ID: 066704. https://doi.org/10.1103/PhysRevE.76.066704

[3] Hashimoto, T., Tanno, I., Morinishi, K. and Satofuka, N. (2012) Simulation of Decaying Two-Dimensional Turbulence Using Kinetically Reduced Local Navier-Stokes Equations. Seventh International Conference on Computational Fluid Dynamics (ICCFD7), Hawaii, 9-13 July 2012, 1-10.

[4] Hashimoto, T., Tanno, I., Yasuda, T., Tanaka, Y., Morinishi, K. and Satofuka, N. (2015) Higher Order Numerical Simulation of Unsteady Viscous Incompressible Flows Using Kinetically Reduced Local Navier-Stokes Equations on a GPU. Computers \& Fluids, 110, 108-113. https://doi.org/10.1016/j.compfluid.2014.09.013

[5] Hashimoto, T., Tanno, I., Yasuda, T., Tanaka, Y., Morinishi, K. and Satofuka, N. (2018) Multi-GPU Parallel Computation of Unsteady Incompressible Flows Using Kinetically Reduced Local Navier-Stokes Equations. Computers \& Fluids, 167, 215-220. https://doi.org/10.1016/j.compfluid.2018.03.028

[6] Sahin, M. and Owens, R.G. (2003) A Novel Fully Implicit Finite Volume Method Applied to the Lid-Driven Cavity Problem-Part I: High Reynolds Number Flow Calculations. International Journal for Numerical Methods in Fluids, 42, 57-77. https://doi.org/10.1002/fld.442

[7] Albensoeder, S. and Kuhlmann, H.C. (2005) Accurate Three-Dimensional Lid-Driven Cavity Flow. Journal of Computational Physics, 206, 536-558.

https://doi.org/10.1016/j.jcp.2004.12.024

[8] Khanafer, K.M., Al-Amiri, A.M. and Pop, I. (2007) Numerical Simulation of Unsteady Mixed Convection in a Driven Cavity Using an Externally Excited Sliding Lid. European Journal of Mechanics-B/ Fluids, 26, 669-687. https://doi.org/10.1016/j.euromechflu.2006.06.006

[9] Cardoso, N. and Bicudo, P. (2008) Time Dependent Simulation of the Driven Lid Cavity at High Reynolds Number. arXiv:0809.3098.

[10] Marchi, C.H., Suero, R. and Araki, L.K. (2009) The Lid-Driven Square Cavity Flow: Numerical Solution with a $1024 \times 1024$ Grid. Journal of the Brazilian Society of Mechanical Sciences and Engineering, 31, 186-198. https://doi.org/10.1590/S1678-58782009000300004

[11] Yu, P.X. and Tian, Z.F. (2013) A Compact stReam Function-Velocity Scheme on Nonuniform Grids for the 2D Steady Incompressible Navier-Stokes Equations. Computers and Mathematics with Applications, 66, 1192-1212. https://doi.org/10.1016/j.camwa.2013.07.013

[12] Ambethkar, V. and Kushawaha, D. (2017) Numerical Simulations of Fluid Flow and Heat Transfer in a Four-Sided, Lid-Driven Rectangular Domain. International Journal of Heat and Technology, 35, 273-278. https://doi.org/10.18280/ijht.350207

[13] Keskin, Y. and Oturanc, G. (2009) Reduced Differential Transform Method for Partial Differential Equations. International Journal of Nonlinear Sciences and Numerical Simulation, 10, 7414-749. https://doi.org/10.1515/IJNSNS.2009.10.6.741

[14] Keskin, Y. and Oturanç, G. (2010) Reduced Differential Transform Method for Generalized KdV Equations. Mathematical and Computational Applications, 15, 382-393. https://doi.org/10.3390/mca15030382

[15] Gupta, P.K. (2011) Approximate Analytical Solutions of Fractional Benney-Lin Equation by Reduced Differential Transform Method and the Homotopy Perturbation Method. Computers and Mathematics with Applications, 61, 2829-2842. https://doi.org/10.1016/j.camwa.2011.03.057

[16] Taghizadeh, N., Akbari, M. and Shahidi, M. (2011) Application of Reduced Differential Transform Method to the Wu-Zhang Equation. Australian Journal of Basic 
and Applied Sciences, 5, 565-571.

[17] Arora, R., Siddiqui, M.J. and Singh, V.P. (2012) Solutions of Inviscid Burgers' and Equal Width Wave Equations by RDTM. International Journal of Applied Physics and Mathematics, 2, 212-214. https://doi.org/10.7763/IJAPM.2012.V2.92

[18] Kolebaje, O.T. and Oyewande, E.O. (2013) Numerical Solution of the (2+1) Dimensional Sine-Gordon Equation by Reduced Differential Transform Method. International Journal of Modern Applied Physics, 2, 15-26.

[19] Abazari, R. and Abazari, M. (2013) Numerical Study of Burgers-Huxley Equations via Reduced Differential Transform Method. Computational and Applied Mathematics, 32, 1-17. https://doi.org/10.1007/s40314-013-0001-2

[20] Srivastava, V.K., Awasthi, M.K. and Kumar, S. (2014) Analytical Approximations of Two and Three Dimensional Time-Fractional Telegraphic Equation by Reduced Differential Transform Method. Egyptian Journal of Basic and Applied Sciences, 1, 60-66. https://doi.org/10.1016/j.ejbas.2014.01.002

[21] Al-Amr, M.O. (2014) New Applications of Reduced Differential Transform Method. Alexandria Engineering Journal, 53, 243-247.

https://doi.org/10.1016/j.aej.2014.01.003

[22] Acan, O. and Keskin, Y. (2015) Reduced Differential Transform Method for $(2+1)$ Dimensional Type of the Zakharov-Kuznetsov ZK(n,n) Equations. AIP Conference Proceedings, 1648, Article ID: 370015. https://doi.org/10.1063/1.4912604

[23] Neog, B.C. (2015) Solutions of Some System of Non-Linear PDEs Using Reduced Differential Transform Method. IOSR Journal of Mathematics, 11, 37-44.

[24] Yu, J., Jing, J., Sun, Y. and Wu, S. (2016) (n+1)-Dimensional Reduced Differential Transform Method for Solving Partial Differential Equations. Applied Mathematics and Computation, 273, 697-705. https://doi.org/10.1016/j.amc.2015.10.016

[25] Gubes, M. and Oturanc, G. (2016) Approximate Solutions of Coupled Ramani Equation by Using RDTM with Compared DTM and Exact Solutions. New Trends in Mathematical Sciences, 4, 198-212. https://doi.org/10.20852/ntmsci.2016.107

[26] Mohamed, M.S. and Gepreel, K.A. (2017) Reduced Differential Transform Method for Nonlinear Integral Member of Kadomtsev-Petviashvili Hierarchy Differential Equations. Journal of the Egyptian Mathematical Society, 25, 1-7. https://doi.org/10.1016/j.joems.2016.04.007

[27] Srivastava, V.K., Awasthi, M.K. and Chaurasia, R.K. (2017) Reduced Differential Transform Method to Solve Two and Three Dimensional Second Order Hyperbolic Telegraph Equations. Journal of King Saud University-Engineering Sciences, 29, 166-171. https://doi.org/10.1016/j.jksues.2014.04.010

[28] Pakdemirli, M. and Boyac, H. (2007) Generation of Root Finding Algorithms via Perturbation Theory and Some Formulas. Applied Mathematics and Computation, 184, 783-788. https://doi.org/10.1016/j.amc.2006.05.207

[29] Aksoy, Y. and Pakdemirli, M. (2010) New Perturbation-Iteration Solutions for Bratu-Type Equations. Computers \& Mathematics with Applications, 59, 2802-2808. https://doi.org/10.1016/j.camwa.2010.01.050

[30] Pakdemirli, M., Aksoy, Y. and Boyac, H. (2011) A New Perturbation-Iteration Approach for First Order Differential Equations. Mathematical and Computational Applications, 16, 890-899.

[31] Aksoy, Y., Pakdemirli, M., Abbasbandy, S. and Boyac, H. (2012) New Perturbation-Iteration Solutions for Nonlinear Heat Transfer Equations. International Journal of Numerical Methods for Heat \& Fluid Flow, 22, 814-828. 
https://doi.org/10.1108/09615531211255725

[32] Șenol, M., Dolapç, İ.T., Aksoy, Y. and Pakdemirli, M. (2013) Perturbation-Iteration Method for First-Order Differential Equations and Systems. Abstract and Applied Analysis, 2013, Article ID: 704137.

[33] Dolapç, İ.T., Șenol, M. and Pakdemirli, M. (2013) New Perturbation Iteration Solutions for Fredholm and Volterra Integral Equations. Journal of Applied Mathematics, 2013, Article ID: 682537.

[34] Khalid, M., Sultana, M., Zaidi, F. and Arshad, U. (2015) An Effective Perturbation Iteration Algorithm for Solving Riccati Differential Equations. International Journal of Computer Applications, 111, 1-5. https://doi.org/10.5120/19571-1361

[35] Aksoy, Y., Goktas, U., Pakdemirli, M. and Dolapç, I.T. (2016) Application of Perturbation-Iteration Method to Lotka-Volterra Equations. Alexandria Engineering Journal, 55, 1661-1666. https://doi.org/10.1016/j.aej.2016.02.015

[36] Șenol, M. and Kasmaei, H.D. (2017) Perturbation-Iteration Algorithm for Systems of Fractional Differential Equations and Convergence Analysis. Progress in Fractional Differentiation and Applications, 4, 271-279. https://doi.org/10.18576/pfda/030403

[37] Akinlabi, G.O. and Edeki, S.O. (2017) Perturbation Iteration Transform Method for the Solution of Newell-Whitehead-Segel Model Equations. Journal of Mathematics and Statistics, 13, 24-29. https://doi.org/10.3844/jmssp.2017.24.29

[38] Șenol, M., Alquran, M. and Kasmaei, H.D. (2018) On the Comparison of Perturbation-Iteration Algorithm and Residual Power Series Method to Solve Fractional Zakharov-Kuznetsov Equation. Results in Physics, 9, 321-327.

https://doi.org/10.1016/j.rinp.2018.02.056

[39] Shih, T.M. and Tan, C.H. (1989) Effects of Grid Staggering on Numerical Schemes. International Journal for Numerical Methods of Fluids, 9, 193-212. https://doi.org/10.1002/fld.1650090206

[40] Pereira, J.M.C., Kobayashi, M.H. and Pereiraz, J.C.F. (2001) A Fourth-Order-Accurate Finite Volume Compact Method for the Incompressible Navier-Stokes Solutions. Journal of Computational Physics, 167, 217-243. https://doi.org/10.1006/jcph.2000.6673

[41] Laizet, S. and Lamballais, E. (2009) High-Order Compact Schemes for Incompressible Flows: A Simple and Efficient Method with Quasi-Spectral Accuracy. Journal of Computational Physics, 228, 5989-6015. https://doi.org/10.1016/j.jcp.2009.05.010

[42] Jotsa, A.C.K. and Pennati, V.A. (2015) A Cost-Effective FE Method for 2D Navier-Stokes Equations. Engineering Applications of Computational Fluid Mechanics, 9, 66-83. https://doi.org/10.1080/19942060.2015.1004811

[43] Yu, P.X., Tian, Z.F. and Zhang, H. (2017) A Rational High-Order Compact Difference Method for the Steady-State Stream Function-Vorticity Formulation of the Navier-Stokes Equations. Computers and Mathematics with Applications, 73, 1461-1484. https://doi.org/10.1016/j.camwa.2017.01.024 